\title{
Currents Induced on the Surface of a Conducting Circular Cylinder by a Slot ${ }^{1}$
}

\author{
Gerard Hasserjian ${ }^{2}$ and $\mathbf{A}$. Ishimaru \\ Contribution from the Department of Electrical Engineering, College of Engineering, \\ University of Washington, Seattle 5, Wash.
}

(Received October 16, 1961)

\begin{abstract}
This paper is a partial study of currents induced on circular, conducting cylinders by narrow radiating slots. First, a brief and general formulation of the radiation fields of slots on cylinders is made. Then, the problem of an infinite axial slot is examined thoroughly for all cylinder sizes. An expansion for the fields, very close to the slot, on large radius cylinders, is also obtained. Sample computations are made, for various ranges of cylinder radius, and the order of the errors is discussed.

The problem of a circumferential slot, of constant excitation, is also considered. An asymptotic expansion obtained for this case yields the surface current distribution for values of axial distances that are smaller than the square of the circumference of the cylinder.

Since one of the objectives of this study is to determine mutual coupling between two slots on a cylinder, the last section presents a formulation of the equivalent network in terms of the surface and feed line currents.
\end{abstract}

\section{Introduction}

The problem of determining the interaction of sources with each other or with other discontinuities, on curved conducting surfaces, is basically a problem of the fields of the sources on the conducting surface. In a previous report Held and Hasserjian [1958] discussed the results of an experimental study showing the effect of the tip of a cone with a slot. The conclusions of that study led us to consider the problem of the fields of a slot on a circular cylinder.

There were several reasons that led us to this approach. Our study of the cone indicated that the cone problem was basically a study of fields on curved conducting surfaces with the cone tip as a singularity. In addition, the mathematical analysis of surface fields on the cone was thought to be a formidable problem. Therefore, a simpler geometrical model was sought which could be studied both theoretically and experimentally and then the results extended to not only the cone but other geometries as well. Therefore, it is believed that a complete study of a circular cylinder will provide fundamental answers to various problems of curved conducting surfaces.

The formal solution of the fields due to sources on cylindrical structures has been treated in the literature [Silver and Saunders, 1950; Sensiper, 1953 and 1957; Wait, 1959]. However, expressions for numerical computations have been limited to the determination of the fields at large distances from the cylinder surface. Several authors have treated the problem of currents on the surface of cylinders [Papas and King, 1949; Lucke, 1953; Nishida, 1960] for infinite axial slots. However, their results do not indicate the magnitude of the errors involved in the approximations that they employ.

In this report we provide expressions, for the surface currents due to infinite axial slots, for all ranges of cylinder radius. The expressions that are used are all asymptotic which provide estimates for the errors. Comparisons are made between the harmonic series and residue series evaluations.

\footnotetext{
1 The research reported in this document has been sponsored by the Electronics Research Directorate of the Air Force Cambridge Research Laboratories, Office of Aerospace Research (USAF), Bedford, Mass. Contract No. AF 19(604)-4098.

${ }^{2}$ Present address of Dr. Gerard Hasserjian: Physics Technology Department, Boeing Company, Seattle, Wash.
} 
The problem of the currents excited by a narrow circumferential slot of constant excitation, running entirely around the cylinder, is also treated. The results are again asymptotic expansions and in this case are limited to axial distances small compared to the circumference of the cylinder.

\section{Formulation of the Cylinder Problem}

In this section we shall formulate the expressions for the fields due to slots located on conducting circular cylinders of infinite length. Such a formulation has been performed by various authors [Silver and Saunders, 1950; Wait, 1959] and is outlined briefly here.

The general solution, for the field components, may be derived from two scalar functions which individually satisfy the wave equation. We shall identify these functions by $\psi_{1}(r, \varphi, Z)$ and $\psi_{2}(r, \varphi, Z)$. The coordinate system and the cylinder orientation is shown in figure 2.1. The formal solution for the two functions may be written

$$
\psi_{1,2}(r, \varphi, Z)=\frac{1}{2 \pi} \int_{-\infty}^{\infty} e^{i \zeta Z} \sum_{n=-\infty}^{\infty} A_{n 1,2} e^{-i n \varphi} H_{n}^{(2)}\left(r \sqrt{k^{2}-\zeta^{2}}\right) d \zeta
$$

where an appropriate path should be chosen for the Fourier inversion. The coefficients of the series, $A_{n 1}$ and $A_{n 2}$, may be determined if the field distribution on a closed surface is known. In this problem, the tangential components of the electric field on the surface of the conducting cylinder will be zero everywhere except across the slot. If the field across the slot is known, then the field distribution on the entire surface of the cylinder is defined, which makes it possible to determine the unknown coefficients of the general solution.

Considering two slot orientations, longitudinal and transverse, and assuming the tangential electric fields to have only components transverse to the slot, we have the following boundary conditions on the surface of the cylinder $(r=a)$. For a longitudinal slot

$$
\begin{aligned}
& E_{Z}=0, \text { for all } Z \text { and } \varphi \\
& E_{\varphi}=f_{1}(\varphi) f_{2}(Z), \text { for } Z_{1} \leq Z \leq Z_{2}, \varphi_{1} \leq \varphi \leq \varphi_{2} \\
& E_{\varphi}=0, \text { everywhere else. }
\end{aligned}
$$

For a transverse slot

$$
\begin{aligned}
& E_{Z}=g_{1}(\varphi) g_{2}(Z), \text { for } Z_{1}^{\prime} \leq Z \leq Z_{2}^{\prime}, \varphi_{1}^{\prime} \leq \varphi \leq \varphi_{2}^{\prime} \\
& E_{Z}=0, \text { everywhere else } \\
& E_{\varphi}=0, \text { for all } Z \text { and } \varphi .
\end{aligned}
$$

These provide sufficient information to determine the coefficients of the general solution and the field components derivable from them.

For a longitudinal slot, the coefficients of the series of $\psi_{2}$ are zero because $E_{Z}=0$. The field components as a function of $\psi_{1}$ are:

$$
\begin{aligned}
& H_{Z}=\frac{i}{\omega \mu \epsilon}\left(\frac{\partial^{2}}{\partial Z^{2}}+k^{2}\right) \psi_{1}, E_{Z}=0 \\
& H_{\varphi}=\frac{i}{\omega \mu \epsilon} \frac{\partial^{2} \psi_{1}}{r \partial \varphi \partial Z}, E_{\varphi}=-\frac{1}{\epsilon} \frac{\partial \psi_{1}}{\partial r} \\
& H_{r}=\frac{i}{\omega \mu \epsilon} \frac{\partial^{2} \psi_{1}}{\partial r \partial Z}, E_{r}=\frac{1}{\epsilon r} \frac{\partial \psi_{1}}{\partial \varphi} .
\end{aligned}
$$

Matching (1) and (2) by the relation $E_{\varphi}=-\frac{1}{\epsilon} \frac{\partial \psi_{1}}{\partial r}$, we find that 
FIGURE 2.1. Coordinates and orientation of the cylinder.
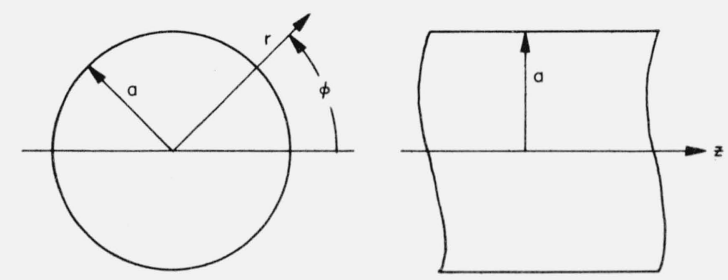

$$
A_{n 1}=\frac{B_{n 1}(\zeta)}{\sqrt{k^{2}-\zeta^{2} H_{n}^{(2)}\left(a \sqrt{k^{2}-\zeta^{2}}\right)}}
$$

where

$$
B_{n 1}(\zeta)=\frac{1}{2 \pi} \int_{Z_{1}}^{Z_{2}} \int_{\varphi_{1}}^{\varphi_{2}} f_{1}(\varphi) f_{2}(Z) e^{i(n \varphi-\zeta Z)} d \varphi d Z
$$

Therefore, for the longitudinal slot

$$
\psi_{1 l}(r, \varphi, Z)=\frac{-\epsilon}{2 \pi} \int_{-\infty}^{\infty} e^{i \zeta Z} \sum_{n=-\infty}^{\infty} \frac{B_{n 1}(\zeta) e^{-i n \varphi} H_{n}^{(2)}\left(r \sqrt{k^{2}-\zeta^{2}}\right)}{\sqrt{k^{2}-\zeta^{2}} H_{n}^{(2) \prime}\left(a \sqrt{k^{2}-\zeta^{2}}\right)} d \zeta .
$$

Once ( 7 ) is evaluated, we can then evaluate the other field components from the rela. tionships in (4).

For a transverse slot, both scalar functions $\psi_{1}$ and $\psi_{2}$ have non-zero coefficients.

The field components as a function of $\psi_{1}$ and $\psi_{2}$ are:

$$
\begin{aligned}
& H_{Z}=\frac{i}{\omega \mu \epsilon}\left(\frac{\partial^{2}}{\partial Z^{2}}+k^{2}\right) \psi_{1 t} \\
& H_{\varphi}=\frac{i}{\omega \mu \epsilon} \frac{\partial^{2} \psi_{1 t}}{r \partial \varphi \partial Z}-\frac{1}{\mu} \frac{\partial \psi_{2}}{\partial r} \\
& H_{r}=\frac{i}{\omega \mu \epsilon} \frac{\partial^{2} \psi_{1 t}}{\partial r \partial Z}+\frac{1}{\mu} \frac{\partial \psi_{2}}{r \partial \varphi} \\
& E_{Z}=\frac{1}{i \omega \mu \epsilon}\left(\frac{\partial^{2}}{\partial Z^{2}}+k^{2}\right) \psi_{2} \\
& E_{\varphi}=-\frac{1}{\epsilon} \frac{\partial \psi_{1 t}}{\partial r}+\frac{1}{i \omega \mu \epsilon} \frac{\partial^{2} \psi_{2}}{r \partial \varphi \partial Z} \\
& E_{r}=\frac{1}{\epsilon} \frac{\partial \psi_{1 t}}{r \partial \varphi}+\frac{1}{i \omega \mu \epsilon} \frac{\partial^{2} \psi_{2}}{\partial r \partial Z} .
\end{aligned}
$$

Using eq (8) and the boundary conditions (3) we obtain from the $E_{Z}$ component

$$
A_{n 2}=\frac{(i \omega \mu \epsilon) B_{n 2}(\zeta)}{\left(k^{2}-\zeta^{2}\right) H_{n}^{(2)}\left(a \sqrt{\left.k^{2}-\zeta^{2}\right)}\right.}
$$

where

$$
B_{n 2}(\zeta)=\frac{1}{2 \pi} \int_{Z_{1}}^{Z_{2}} \int_{\varphi_{1}^{\prime}}^{\varphi_{2}^{\prime}} g_{1}(\varphi) g_{2}(Z) e^{i(n \varphi-\zeta Z)} d \varphi
$$

Also, from $E_{\varphi}$ we have

$$
A_{n 1}=\frac{n \zeta}{i \omega \mu a} \frac{H_{n}^{(2)}\left(a \sqrt{k^{2}-\zeta^{2}}\right) A_{n 2}}{\sqrt{k^{2}-\zeta^{2}} H_{n}^{(2) \prime}\left(a \sqrt{k^{2}-\zeta^{2}}\right)}
$$


or

$$
A_{n 1}=\left(\frac{\epsilon}{a}\right) \frac{n \zeta B_{n 2}(\zeta)}{\left(k^{2}-\zeta^{2}\right)^{3 / 2} H_{n}^{(2) \prime}\left(a \sqrt{k^{2}-\zeta^{2}}\right)} .
$$

Therefore, for the scalar function $\psi_{1 t}$ of the transverse slot, we have

and

$$
\psi_{1 t}(r, \varphi, Z)=\frac{\epsilon}{2 \pi a} \int_{-\infty}^{\infty} e^{i \zeta Z} \sum_{n=-\infty}^{\infty} \frac{n \zeta B_{n 2}(\zeta) e^{-i n \varphi} H_{n}^{(2)}\left(r \sqrt{k^{2}-\zeta^{2}}\right)}{\left(k^{2}-\zeta^{2}\right)^{3 / 2} H_{n}^{(2) \prime}\left(a \sqrt{k^{2}-\zeta^{2}}\right)} d \zeta
$$

$$
\psi_{2 t}(r, \varphi, Z)=\frac{i \omega \mu \epsilon}{2 \pi} \int_{-\infty}^{\infty} e^{i \zeta Z} \sum_{n=-\infty}^{\infty} \frac{B_{n 2}(\zeta) e^{-i n \varphi} H_{n}^{(2)}\left(r \sqrt{k^{2}-\zeta^{2}}\right)}{\left(k^{2}-\zeta^{2}\right) H_{n}^{(2)}\left(a \sqrt{k^{2}--\zeta^{2}}\right)} d \zeta .
$$

Substitution of (13) and (14) in (8) will yield the desired field components at" any point around the cylinder.

In the expressions of (7), (13), and (14) we have integrals of the form

$$
I_{n}(Z)=\int_{-\infty}^{\infty} e^{i \zeta Z} f_{n}(\zeta) H_{n}^{(2)}\left(r \sqrt{k^{2}-\zeta^{2}}\right) d \zeta
$$

where the path of integration should be specified around the singularities of the integrand. These singularities are two branch points located at $\zeta= \pm k$.

For the Hankel function of the second kind, it is necessary to require that over the path of integration of (15)

$$
-(\pi+\epsilon)<\arg \sqrt{k^{2}-\zeta^{2}}<\epsilon .
$$

Furthermore, in order to have outward radial propagation and to keep the fields bounded at infinity, it is necessary to require that for $k$ complex

$$
\operatorname{Re}\left[\sqrt{k^{2}-\zeta^{2}}\right] \geq 0, \operatorname{Im}\left[\sqrt{k^{2}-\zeta^{2}}\right] \leq 0 .
$$

For $k$ real, (17) reduces to

$$
\begin{gathered}
\arg \sqrt{k^{2}-\zeta^{2}}=0, \text { for }|\zeta|<k \\
\arg \sqrt{k^{2}-\zeta^{2}}=-\frac{\pi}{2} \text {, for }|\zeta|>k
\end{gathered}
$$

Therefore, in discussions of the integral in (15), we shall denote the path of integration by $\mathrm{C}_{1}$, as shown in figure 2.2. The branch cuts and the path of integration conform with the constraints specified by (16), (17), and (18). When $k$ is real, the path of integration should avoid the singularity as shown by the dotted curve in the figure.

This completes the formulation of the fields of slots on conducting cylinders. In the sections that follow, we shall evaluate the magnetic field on the surface of the cylinder for a uniformly excited longitudinal slot of infinite length and a circumferential slot running entirely around the cylinder.

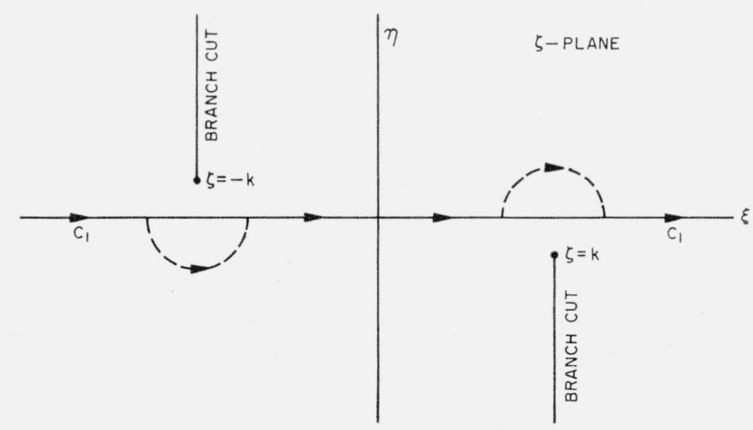

Figure 2.2. Path of integration in the $\zeta$-plane. 


\section{Infinite Axial Slot With Constant Excitation}

A longitudinal slot, of narrow width, can be said to have a constant electric field across the slot. Therefore, on the surface of the cylinder

$$
E_{\varphi}=\frac{V_{0}}{2 a \psi_{0}}\left[U\left(\varphi+\psi_{0}\right)-U\left(\varphi-\psi_{0}\right)\right] \int_{-\infty}^{\infty} f\left(Z^{\prime}\right) \delta\left(Z-Z^{\prime}\right) d Z^{\prime}
$$

where $V_{0}$ is the voltage across the slot at some reference point, $2 \psi_{0}$ is the angular width, $a$ is the radius of the cylinder, $f(Z)$ is the axial variation of the voltage along the slot and the functions inside the brackets are the usual Heaviside, step functions. Since the integral implies the summation of infinitesimal slots, for the purpose of formulating the problem, we shall first assume

$$
f(Z)=\delta\left(Z-Z^{\prime}\right) .
$$

Therefore, considering (1) and (2) and evaluating the coefficients $B_{n 1}(\zeta)$ defined in $(2-6)$, we have

$$
B_{n 1}(\zeta)=\frac{V_{0}}{2 \pi a} \frac{\sin n \psi_{0}}{n \psi_{0}} e^{-i \zeta Z^{\prime}}
$$

Substituting (3) in $(2-7)$, we have

$$
\psi_{1 l}(r, \varphi, Z)=\frac{-1}{2 \pi} \frac{V_{0} \epsilon}{2 \pi a} \sum_{n=-\infty}^{\infty} \frac{\sin n \psi_{0}}{n \psi_{0}} e^{-i n \varphi} \int_{-\infty}^{\infty} \frac{e^{i \zeta\left(Z-Z^{\prime}\right)} H_{n}^{(2)}\left(r \sqrt{k^{2}-\zeta^{2}}\right) d \zeta}{\sqrt{k^{2}-\zeta^{2}} H_{n}^{(2) \prime}\left(a \sqrt{k^{2}-\zeta^{2}}\right)} .
$$

In order to evaluate the fields due to any slot with an excitation $f(Z)$ along its axis, we need to integrate the product of $f\left(Z^{\prime}\right)$ and (4) with respect to $Z^{\prime}$.

To obtain the appropriate expression for the longitudinal slot of infinite extent, of $2 a \psi_{0}$ width and of constant excitation along the $Z$-axis, we integrate eq (4) with respect to $Z^{\prime}$ from $-\infty$ to $+\infty$. Since

then (4) becomes

$$
\frac{1}{2 \pi} \int_{-\infty}^{\infty} e^{i \zeta\left(Z-Z^{\prime}\right)} d Z^{\prime}=-\delta(\zeta)
$$

$$
\psi_{1 l}(r, \varphi)=+\frac{V_{0} \epsilon}{2 \pi k a} \sum_{n=-\infty}^{\infty} \frac{\sin n \psi_{0}}{n \psi_{0}} e^{-i n \varphi} \frac{H_{n}^{(2)}(k r)}{H_{n}^{(2)}(k a)} .
$$

From this result expressions for any of the field components can be obtained. However, we shall concern ourselves only with the numerical evaluation of the magnetic field on the surface of the cylinder.

In order to obtain expressions which are amenable to computations, it is necessary to consider approximations valid in different ranges of cylinder radius. We will obtain approximations valid in the ranges $k a<<1, k a \leq 25$ and $k a \geq 10$.

\subsection{Small Radius Cylinders}

The scalar function (6) evaluated on the surface of the cylinder is

$$
\psi_{1}(a, \varphi)=\frac{-V_{0} \epsilon}{\pi}\left[\frac{H_{0}^{(2)}(k a)}{2 k a H_{1}^{(2)}(k a)}-\sum_{n=1}^{\infty} \frac{\sin n \psi_{0}}{n \psi_{0}} \frac{\cos n \varphi}{k a} \frac{H_{n}^{(2)}(k a)}{H_{n}^{(2) \prime}(k a)}\right]
$$

for $n \geq 1$ and $k a<<1$

$$
\frac{H_{n}^{(2)}(k a)}{H_{n}^{(2)}(k a)} \cong-\left(\frac{k a}{n}\right)\left[1+0\left[(k a)^{2}\right]\right] .
$$

Therefore,

$$
\psi_{1}(a, \varphi) \cong-\frac{V_{0} \epsilon}{\pi}\left[\frac{H_{0}^{(2)}(k a)}{2 k a H_{1}^{(2)}(k a)}+\sum_{n=1}^{\infty} \frac{\sin n \psi_{0} \cos n \varphi}{\psi_{0} n^{2}}\right]+0\left[(k a)^{2}\right]
$$


The sum of the infinite series in (9)

$$
S_{1}(\varphi)=\sum_{n=1}^{\infty} \frac{\sin n \psi_{0} \cos n \varphi}{\psi_{0} n^{2}}
$$

is a symmetric function of $\varphi$ and is evaluated in appendix I. Therefore,

$$
\psi_{1}(a, \varphi) \cong-\frac{V_{0} \epsilon}{\pi}\left[\frac{H_{0}^{(2)}(k a)}{2 k a H_{1}^{(2)}(k a)}+S_{1}(\varphi)\right]
$$

using (2 to 7 ) and (11), the magnetic field on the surface of the cylinder becomes

$$
H_{\varphi} \cong \frac{V_{0}}{i \omega \mu \pi}\left[\frac{H_{0}^{(2)}(k a)}{2 k a H_{1}^{(2)}(k a)}+S_{1}(\varphi)\right]
$$

where for $\psi_{0} \leq \varphi \leq \pi$

$$
\begin{aligned}
S_{1}(\varphi)=1 & +\left(\frac{\tan (\varphi / 2)}{\psi_{0}}-\frac{1}{2}\right) \ln \left(2 \sin (\varphi / 2)-\psi_{0} \cos (\varphi / 2)\right) \\
& -\left(\frac{\tan (\varphi / 2)}{\psi_{0}}+\frac{1}{2}\right) \ln \left(2 \sin (\varphi / 2)+\psi_{0} \cos (\varphi / 2)\right)
\end{aligned}
$$

Appendix I also includes the evaluation of $S_{1}$ for $-\psi_{0} \leq \varphi \leq \psi_{0}$, making it possible to evaluate the magnetic field across the slot. Figure $3.1 \mathrm{~A}$ shows the plot of sample computations for the magnetic field distribution on the surface of cylinders of $k a<<1$ and $\psi_{0}=1 / 40$, and figure $3.1 \mathrm{~B}$ is an expanded plot of the magnetic field in the region of the slot for various values of slot widths $2 \psi_{0}$. Both figures represent the plot of the bracketed term in (12).

$$
\psi_{1}^{\prime}(\varphi)=\frac{H_{0}^{(2)}(k a)}{2 k a H_{1}^{(2)}(k a)}+S_{1}(\varphi)
$$

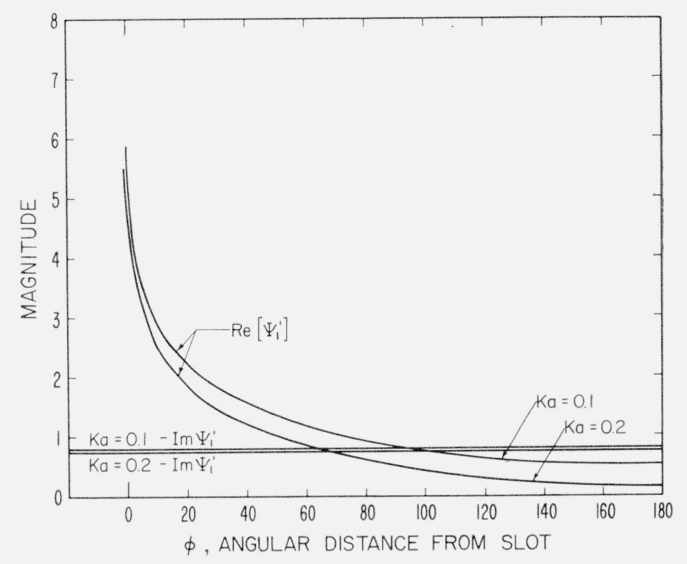

FIgURE 3.1A. Plot of $\psi_{1}^{\prime}=\frac{\mathrm{H}_{0}^{(2)}(\mathrm{ka})}{2 \mathrm{ka} \mathrm{H}_{1}^{(2)}(\mathrm{ka})}+\mathrm{S}_{1}(\varphi)$.

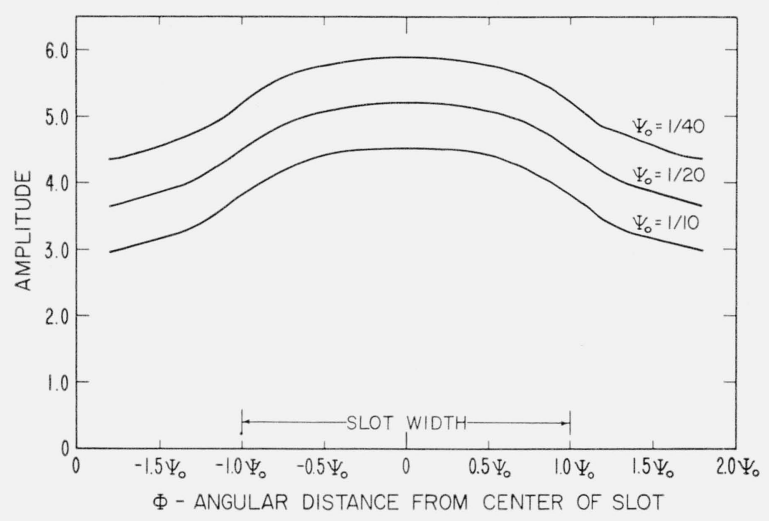

Figure 3.1B. The expanded plot of $\psi_{1}^{\prime}(\varphi)$ around slot for $\mathrm{ka}=0.1$.

\subsection{Intermediate Range Cylinders}

For cylinders having radii $0 \leq k a \leq 25$ (this also includes the first range), the numerical evaluation is obtained by approximating the Hankel functions for large orders and using the approximation for the higher indexed terms of the series. A paper by C. H. Papas and R. King. [1949] shows this evaluation without indicating the order of the errors involved. In this 
section we attempt to bridge this gap. Furthermore, the results obtained in this analysis can be employed to determine the magnetic field across the slot of widths up to $30^{\circ}$ with great ease, making it possible to evaluate the slot impedance. The examples, considered in the analysis, are assumed to have a constant electric field across the slot. However, the analysis can be applied to other field distributions, provided the axial distribution is constant.

Taking the expression in (7) and letting $k a=\rho$, we write

$$
\psi_{1}(\rho, \varphi)=\frac{-V_{0} \epsilon}{\pi}\left[\frac{H_{0}^{(2)}(\rho)}{2 \rho H_{1}^{(2)}(\rho)}-\sum_{n=1}^{\infty} \frac{\sin n \psi_{0}}{n \psi_{0}} \frac{\cos n \varphi}{\rho} \frac{H_{n}^{(2)}(\rho)}{H_{n}^{(2)}(\rho)}\right] .
$$

For $n\left[1-\left(\frac{\rho}{n}\right)^{2}\right]^{1 / 2}>>1$ and $\frac{n}{\rho}=\cosh \alpha$, we have (see [Watson, 1952] page 243)

$$
\begin{aligned}
& H_{n}^{(2)}(\rho) \cong i \sqrt{\frac{2}{\pi \rho \sinh \alpha}} e^{\rho(\alpha \cosh \alpha-\sinh \alpha)}\left[1+0\left(\frac{1}{\rho \sinh \alpha}\right)\right] \\
& H_{n}^{(2) \prime}(\rho) \simeq-i \sqrt{\frac{2 \sinh \alpha}{\pi \rho}} e^{\rho(\alpha \cosh \alpha-\sinh \alpha)}\left[1+0\left(\frac{1}{\rho \sinh \alpha}\right)\right] .
\end{aligned}
$$

Therefore, the ratio of the two functions above becomes

$$
\frac{H_{n}^{(2)}(\rho)}{H_{n}^{(2) \prime}(\rho)} \cong-\frac{1}{\sinh \alpha}\left[1+0\left(\frac{1}{\rho \sinh \alpha}\right)\right] \cong-\left(\frac{\rho}{n}\right)\left[1+\frac{1}{2}\left(\frac{\rho}{n}\right)^{2}+0\left[\frac{1}{n}+\left(\frac{\rho}{n}\right)^{4}\right]\right] .
$$

This leads to an approximate expression for the series in (14), which can now be expressed as

$$
S \cong \sum_{n=1}^{M-1} \frac{\sin n \psi_{0}}{n \psi_{0}} \frac{\cos n \varphi}{\rho} \frac{H_{n}^{(2)}(\rho)}{H_{n}^{(2) \prime}(\rho)}-\sum_{n=M}^{\infty} \frac{\sin n \psi_{0}}{n \psi_{0}} \frac{\cos n \varphi}{n}-\frac{\rho^{2}}{2} \sum_{n=M}^{\infty} \frac{\sin n \psi_{0}}{n \psi_{0}} \frac{\cos n \varphi}{n^{3}}+R_{M}^{\prime}
$$

where, for sufficiently large $M$

$$
R_{M}^{\prime} \leq \sum_{n=M}^{\infty} \frac{\cos n \varphi}{n^{2}}+\rho^{4} \sum_{n=M}^{\infty} \frac{\cos n \varphi}{n^{5}}
$$

Considering, now, the series

$$
s=\sum_{n=M}^{\infty} \frac{\sin n \psi_{0}}{n \psi_{0}} \frac{\cos n \varphi}{n^{3}}
$$

appearing in (17), we write

$$
s=\frac{1}{M} \sum_{n=M}^{\infty} \frac{\sin n \psi_{0}}{n \psi_{0}} \frac{\cos n \varphi}{n^{2}}+R_{M}^{\prime \prime}
$$

where, for sufficiently large $M$

$$
R_{M}^{\prime \prime} \leq \sum_{n=M+1}^{\infty}\left(\frac{1}{n^{3}}-\frac{1}{M n^{2}}\right) \cos n \varphi
$$

In order to evaluate the upper bounds of (18) and (20), we employ the Euler-Maclaurin sum formula. The members of the series in (18) and (20) have the form

$$
f_{k}(n)=\frac{\cos \varphi n}{n^{k}} .
$$

Therefore, with $B_{p}$ and $\varphi_{p}(t)$ as the Bernoulli constants and polynomials respectively, we have from Whittaker and Watson [1952, p. 128], 


$$
\sum_{n=M}^{\infty} f_{k}(n)=\int_{M}^{\infty} f_{k}(x) d x+\frac{1}{2} f_{k}(M)+\sum_{p=1}^{m-1} \frac{(-1)^{p} B_{p}}{(2 p) !} f_{k}^{2 p-1}(M)+R_{m}
$$

where

$$
R_{m}=\frac{1}{(2 m) !} \int_{0}^{1} \varphi_{2 m}(t)\left[\sum_{p=0}^{\infty} f_{k}^{2 m}(M+p+t)\right] d t .
$$

Considering (21) in (22), it is immediately apparent that the dominant term of (22) will be

$$
I_{1}=\int_{M}^{\infty} \frac{\cos \varphi x}{x^{k}} d x
$$

Integrating this by parts, we have

$$
I_{1}=-\frac{\sin M \varphi}{\varphi M^{k}}+\frac{k}{\varphi} \int_{M}^{\infty} \frac{\sin x \varphi}{x^{k+1}} d x
$$

Furthermore,

$$
\begin{aligned}
\frac{1}{\varphi} \int_{M}^{\infty} \frac{\sin x \varphi}{x^{k+1}} d x & \leq \frac{1}{M^{k-1}} \text { for } M_{\varphi} \text { small } \\
& \leq \frac{1}{\varphi M^{k}} \text { for } M_{\varphi} \text { large. }
\end{aligned}
$$

Therefore, for a sufficiently large $M$, we can write

$$
I_{1}=0\left[\left\langle\frac{\sin M \varphi}{\varphi M^{k}}\right\rangle\right]
$$

where by the symbol, $\langle>$, we mean the bounds described above.

This leads to the conclusion that, for a sufficiently large $M$

$$
\sum_{n=M}^{\infty} \frac{\cos n \varphi}{n^{k}}=0\left[\left\langle\frac{\sin M \varphi}{\varphi M^{k}}\right\rangle\right]
$$

We can now use (23) to estimate the upper bounds of (18) and (20). Therefore, the expression in (17) becomes

$$
S \cong \sum_{n=1}^{M-1} \frac{\sin n \psi_{0}}{n \psi_{0}} \cos n \varphi\left[\frac{H_{n}^{(2)}(\rho)}{\rho H_{n}^{(2) \prime}(\rho)}+\frac{1}{n}+\frac{1}{2 M}\left(\frac{\rho}{2 n}\right)^{2}\right]-\left[S_{1}+\frac{\rho^{2}}{2 M} S_{2}\right]+R_{M}
$$

where

$$
\begin{aligned}
S_{1} & =\sum_{n=1}^{\infty} \frac{\sin n \psi_{0}}{n \psi_{0}} \frac{\cos n \varphi}{n} \\
S_{2} & =\sum_{n=1}^{\infty} \frac{\sin n \psi_{0}}{n \psi_{0}} \frac{\cos n \varphi}{n^{2}} \\
R_{M} & =0\left(\left\langle\frac{\sin M \varphi}{M \varphi}\right\rangle\left[\frac{1}{M}+\frac{1}{M}\left(\frac{\rho}{M}\right)^{2}+\left(\frac{\rho}{M}\right)^{4}\right]\right) .
\end{aligned}
$$

The infinite series $S_{1}$ and $S_{2}$ can be expressed in a closed form and are evaluated in appendix I. The dominant term in the expression for $R_{M}$ depends on the values of $M, \varphi$, and $\rho$.

Therefore, the magnetic field on the surface of the cylinder is

$$
H_{Z}=\frac{i k^{2}}{\omega \mu \epsilon} \psi_{1}(\rho, \varphi) \cong \frac{i \omega V_{0}}{\pi}\left(\frac{H_{0}^{(2)}(k a)}{2 k a H_{1}^{(2)}(k a)}-S^{1}+S_{1}+\frac{k a}{2 M} S_{2}\right)
$$


where

$$
\begin{aligned}
& S^{1}=\sum_{n=1}^{M-1} \frac{\sin n \psi_{0}}{n \psi_{0}} \cos n \varphi\left[\frac{H_{n}^{(2)}(k a)}{2 k a H_{n}^{(2) \prime}(k a)}+\frac{1}{n}+\frac{1}{2 M}\left(\frac{k a}{n}\right)^{2}\right] \\
& S_{1}=1+\left(\frac{\tan \varphi / 2}{\psi_{0}}-\frac{1}{2}\right) \ln \left(2 \sin \frac{\varphi}{2}-\psi_{0} \cos \frac{\varphi}{2}\right)-\left(\frac{\tan \varphi / 2}{\psi_{0}}+\frac{1}{2}\right) \ln \left(2 \sin \frac{\varphi}{2}-\psi_{0} \cos \frac{\varphi}{2}\right)
\end{aligned}
$$

for $\psi_{0} \leq \varphi \leq \pi$, and

$$
S_{2}=\frac{\pi^{2}}{6}-\frac{\pi}{2} \varphi+\frac{1}{4} \varphi^{2}+\frac{1}{12} \psi_{0}^{2}
$$

The order of the error of (25) can be estimated from (24). Figures 3.2, 3.3, 3.4, and 3.5 are sample computations of the bracketed term in (25), which are identified by $\psi_{1}$. These computations were performed by a digital computer and the following conditions were imposed.

$$
M=10 \text { for } 0<k a \leq 3
$$

and

$$
M=3[k a] \text { for } 3<k a \leq 25 \text {. }
$$

By $[k a]$ we mean the integer following $k a$.

The results in this section show clearly the orders of the approximations used in computing the magnetic field on the suriace of the cylinder by using finite terms of the harmonic series. Even though one example of the slot angular width $\left(2 \psi_{0}=1 / 20\right)$ was considered, the expressions are valid for widths up to $\psi_{0}=1 / 2$. In addition, for values of $k a$ large, fairly accurate values for the surface fields near and across the slot can be obtained.

These results can also be compared with the residue series evaluations since computations are not restricted to small values of $k a$. Such a comparison is made in section 3.4.

In computing the examples in the figures, the Hankel functions for $S^{1}$ (see eq (25)) were computed by using the recursion formulas. For the real part of the Hankel function, $J_{n}(\rho)$, the iterative steps were started at index $M$ and $M-1$, and for the imaginary part of the Hankel function, $Y_{n}(\rho)$, the iteration was begun from $n=0$, 1 . This approach reduces possible cumulative errors due to the iteration process.

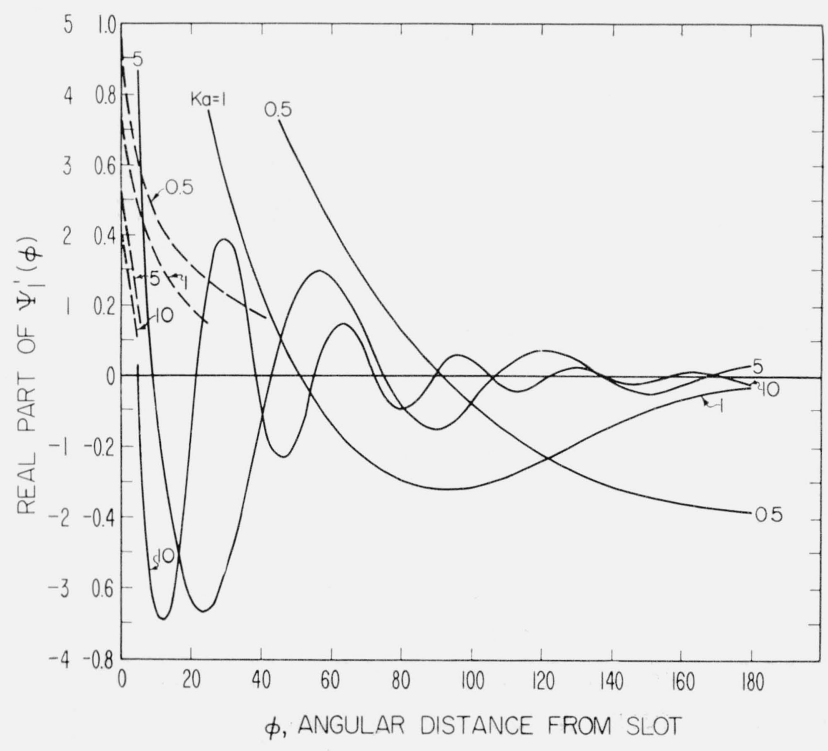

Figure 3.2. Imaginary part of the magnetic field on the cylinder surface, for $k a=0.5,1.0,5.0,10$, $\psi_{0}=1 / 40$.

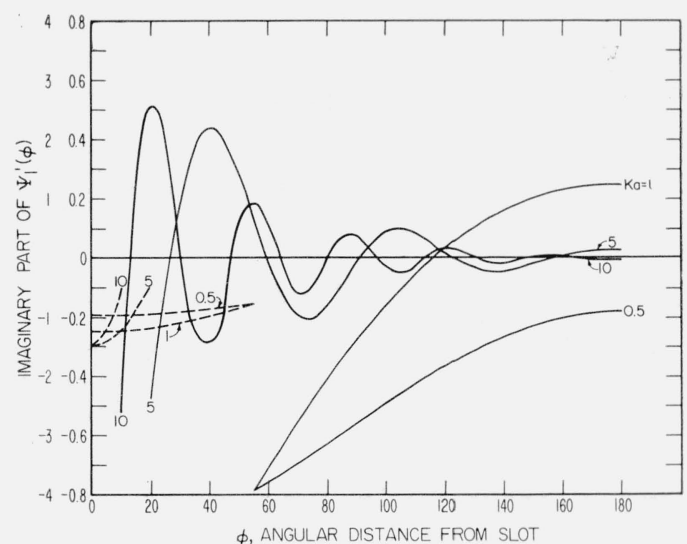

Figure 3.3. Real part of the magnetic field on the cylinder surface, for $k a=0.5,1.0,5.0,10, \psi_{0}=1 / 40$. 


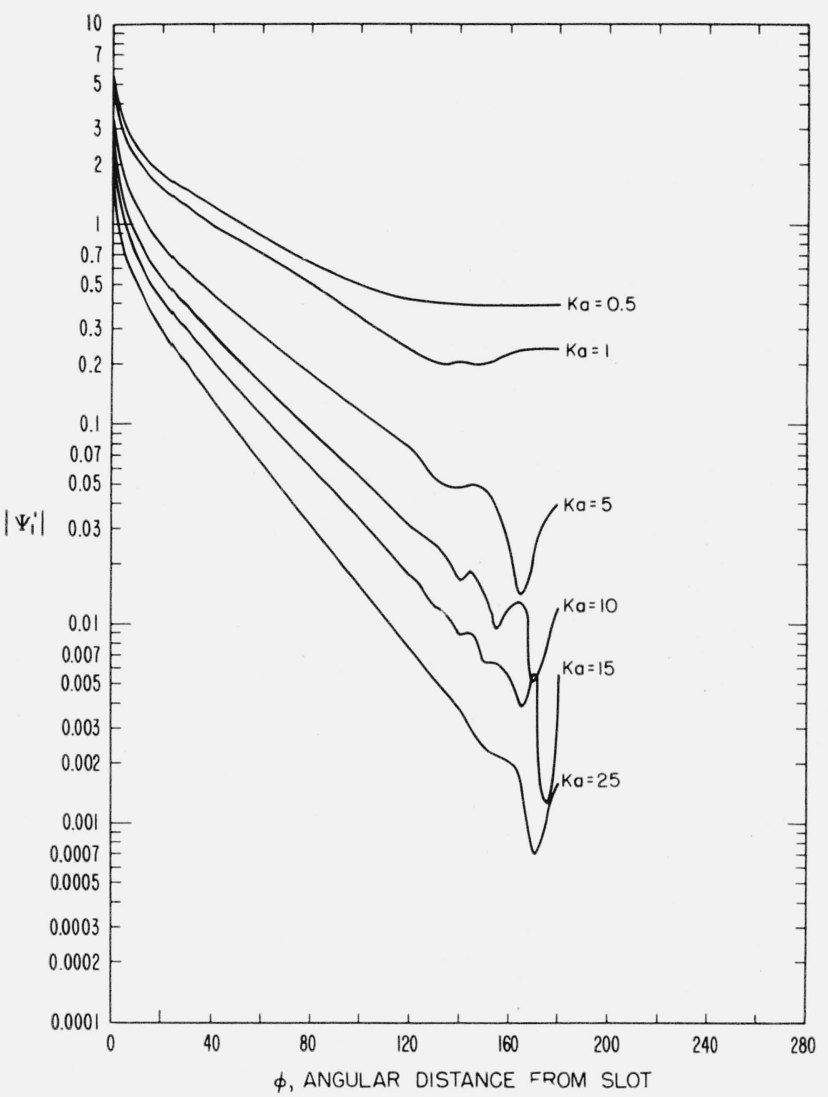

FIG URE 3.4. Magnitude of the magnetic field on the cylinder surface.

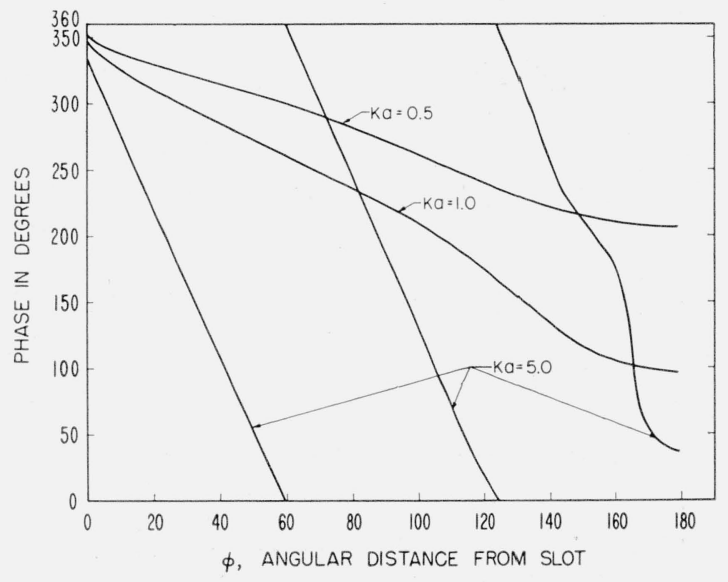

Figure 3.5A. Phase of the magnetic field $\left(k_{v} a=0.5\right.$, $1.0,5.0)$.

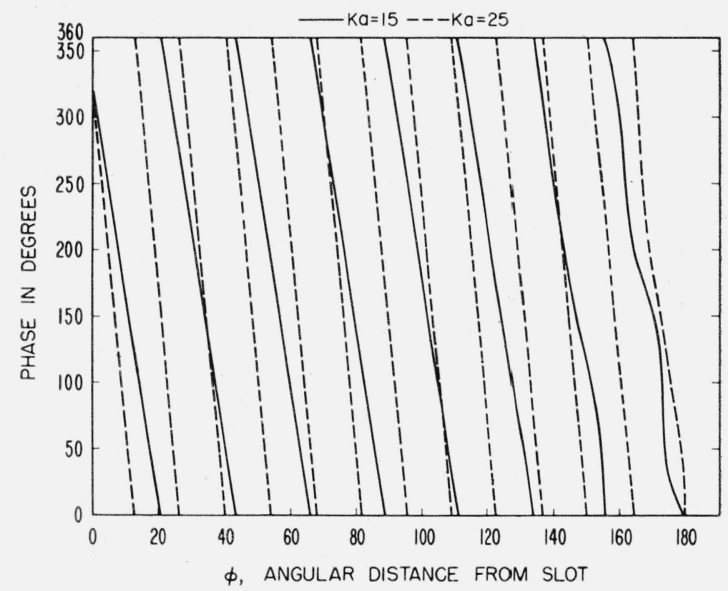

Figure 3.5B. Phase of the magnetic field $(k a=15$, 25).

\subsection{Large Radius Cylinders}

For cylinders having radii in the range $k a \geq 10$, the standard Watson Transformation [Bremmer, 1949; Sensiper, 1957; Wait, 1959] can be used quite adequately. The expression

$$
\psi_{1}(a, \varphi)=-\frac{V_{0} \epsilon}{2 \pi k a} \sum_{n=-\infty}^{\infty} \frac{\sin n \psi_{0}}{n \psi_{0}} e^{-i n \varphi} \frac{H_{n}^{(2)}(k a)}{H_{n}^{(2) \prime}(k a)}
$$

is a residue series of the contour integral

$$
\psi_{1}(a, \varphi)=+\frac{V_{0} \epsilon}{2 \pi k a}\left[\frac{1}{2 i} \int_{C_{1}^{\prime}} \frac{\sin v \psi_{0}}{v \psi_{0}} \frac{e^{-i v(\varphi-\pi)}}{\sin v \pi} \frac{H_{v}^{(2)}(k a)}{H_{v}^{(2) \prime}(k a)} d v\right]
$$

where $C_{1}^{\prime}$ is specified in the $v$-plane as in figure 3.6. The series in (26) represents the sum of the residues of the poles of the integrand in (27) that occur on the real axis. However, the contour $C_{1}^{\prime}$ can be deformed into a new contour $C_{2}^{\prime}$, which encloses the zeroes of $H_{v}^{(2) \prime}(k a)$ providing another residue series. Figure 3.6 also shows the deformed contour $C_{2}^{\prime}$ which encloses the poles, $v_{m}$. The poles $v_{m}$ are the set of roots of

$$
H_{v}^{(2) \prime}(k a)=0
$$




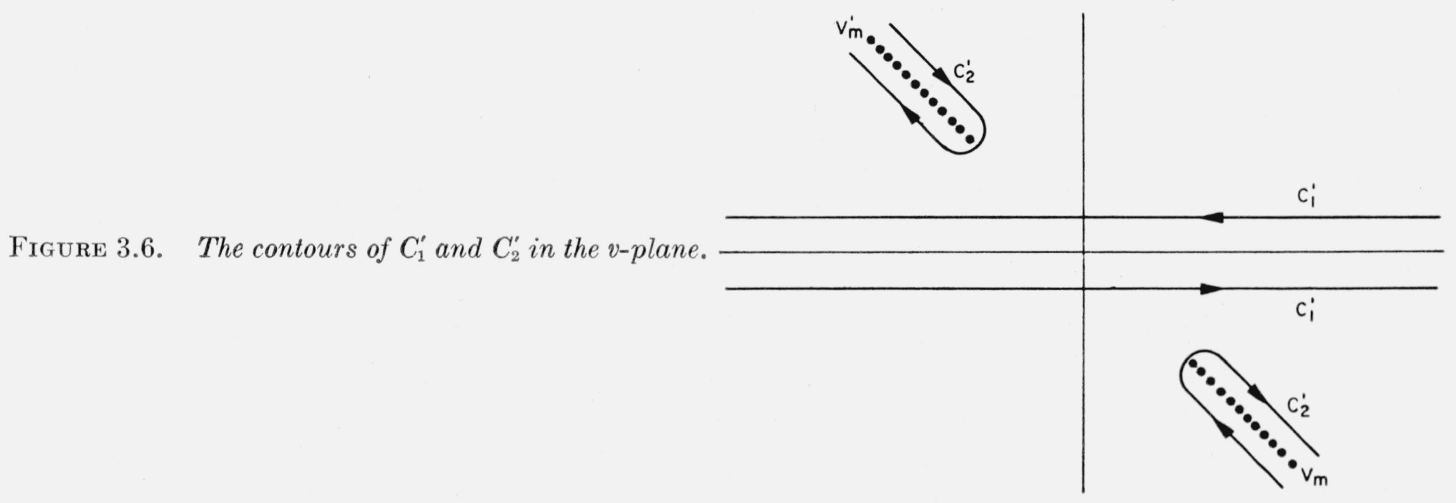

identified by the index $m=1,2, \ldots$. This new series converges rapidly for $\varphi$ and $k a$ large. However, it will be necessary to evaluate the integral (27), by another method, for small values of $\varphi$.

We first take the residue series about the poles $v_{m}$. Since

$$
H_{v}^{(2) \prime}(k a)=e^{-i v \pi} H_{-v}^{(2) \prime}(k a),
$$

and if the zeroes of $H_{v}^{(2) \prime}(k a)$ are $v_{m}$ in the lower half plane, then the poles in the upper half plane are $v_{m}^{\prime}=-v_{m}$. Therefore, the integral in (27) expressed as a residue series is

$$
\begin{aligned}
\psi_{1}(a, \varphi)=-\frac{V_{0} \epsilon}{2 k a}\left[\sum_{m=1}^{\infty} \frac{\sin v_{m}^{\prime} \psi_{0}}{v_{m}^{\prime} \psi_{0}} \frac{e^{-i v_{m}^{\prime}(\varphi-\pi)}}{\sin v_{m}^{\prime} \pi}\left(\frac{H_{v}^{(2)}(k a)}{\partial v} H_{v}^{(2) \prime}(k a)\right)_{v=v_{m}^{\prime}}\right. \\
\left.+\sum_{m=1}^{\infty} \frac{\sin v_{m} \psi_{0}}{v_{m} \psi_{0}} \frac{e^{-i v_{m}(\varphi-\pi)}}{\sin v_{m} \pi}\left(\frac{H_{v}^{(2)}(k a)}{\frac{\partial}{\partial v} H_{v}^{(2) \prime}(k a)}\right)_{v=v_{m}}\right] \\
=-\frac{V_{0} \epsilon}{k a} \sum_{m=1}^{\infty} \frac{\sin V_{m} \psi_{0}}{v_{m} \psi_{0}} \frac{\cos v_{m}(\varphi-\pi)}{\sin v_{m} \pi}\left[\frac{H_{v}^{(2)}(k a)}{\frac{\partial}{\partial v} H_{v}^{(2) \prime}(k a)}\right]_{v=v_{m}}
\end{aligned}
$$

Note the sign change due to the clockwise rotation of the contour $C_{2}^{\prime}$. Equation (28) expresses the fields on the cylinder surface as attenuated standing waves in the $\varphi$-direction. It is also possible to express the fields in terms of traveling waves. We take

$$
\frac{1}{\sin v \pi}=\mp 2 i \sum_{p=0} e^{ \pm i o(2 p+1) \pi}
$$

as a function of the complex variable $v$. The first sign represents the value on the upper half plane and the second sign represents the value on the lower half plane. Considering the two parts of (28) and identifying $\psi_{1}^{-}$and $\psi_{1}^{+}$as the two sums representing the residues in the upper and lower half planes respectively, we have

$$
\begin{aligned}
& \psi_{1}^{-}=+\frac{i V_{0} \epsilon}{k a} \sum_{p=0}^{\infty} \sum_{m=1}^{\infty} R_{1} \frac{\sin v_{m}^{\prime} \psi_{0}}{v_{m}^{\prime} \psi_{0}} e^{+i v_{m}^{\prime}[2(p+1) \pi-\varphi]} \\
& \psi_{1}^{+}=-\frac{i V_{0} \epsilon}{k a} \sum_{p=0}^{\infty} \sum_{m=1}^{\infty} R_{1} \frac{\sin v_{m} \psi_{0}}{v_{m} \psi_{0}} e^{-i v_{m}[2 p \pi+\varphi]}
\end{aligned}
$$

where $R_{1}$ represents the ratio containing the Hankel functions. 
The $v_{m}$ 's, which are roots of $H_{v}^{(2) '}(k a)=0$, fall in the fourth quadrant of the $v$-plane. Therefore, one could say that $(30 \mathrm{~b})$ represents the waves traveling in the positive $\varphi$ direction and that the integers, $p$, represent the waves of mode $m$ that have encircled the cylinder $p$ times. For large cylinders the $p=0$ term would be sufficient to represent the field since waves that have completely gone around the cylinder will be highly attenuated.

To evaluate $R_{1}$, the ratio containing the Hankel functions, we use the approximation for the Hankel function discussed in appendix II.

We need to evaluate

$$
R_{1}=\left.\frac{H_{v}^{(2)}(k a)}{\frac{\partial}{\partial v} H_{v}^{(2) \prime}(k a)}\right|_{v=v_{m}}
$$

where the $v_{m}$ 's are the roots of $H_{r}^{(2) \prime}(k a)=0$.

From (11) and (12) of appendix II we have

$$
H_{v}^{(2)}(Z) \cong-\frac{1}{i \sqrt{\pi}}\left(\frac{2}{Z}\right)^{1 / 3} \omega(t)
$$

and

$$
H_{v}^{(2) '}(Z) \cong \frac{1}{i \sqrt{\pi}}\left(\frac{2}{Z}\right)^{2 / 3} \omega^{\prime}(t)
$$

where

$$
v=Z+\left(\frac{Z}{2}\right)^{1 / 3} t
$$

If we let $Z=k a$ and substitute the above approximations into (31), we have

$$
R_{1} \simeq-\left.\left(\frac{2}{Z}\right)^{-1 / 3} \frac{\omega(t)}{\frac{\partial}{\partial t} \omega^{\prime}(t)} \frac{\partial v}{\partial t}\right|_{v=v_{m}}=-\left(\frac{Z}{2}\right)^{2 / 3} \frac{\omega\left(t_{m}\right)}{\omega^{\prime \prime}\left(t_{m}\right)}
$$

where the $t_{m}$ 's are the roots of $\omega^{\prime}(t)=0$ corresponding to the $v_{m}$ 's by the above relationships.

From the differential equation of the function $\omega(t)$, discussed in appendix II, we have

$$
\omega^{\prime \prime}(t)=t \omega(t) .
$$

Substitution of (33) in (32) leads to

$$
R_{1} \cong-\left(\frac{Z}{2}\right)^{2 / 3} \frac{1}{t_{m}}
$$

Substituting this result in $(30), v_{m}$ for $-v_{m}^{\prime}$ and taking the $p=0$ term we have

$$
\begin{aligned}
& \psi_{1}^{-}=+i\left(\frac{V_{0} \epsilon}{k a}\right)\left(\frac{k a}{2}\right)^{2 / 3} \sum_{m=1}^{\infty} \frac{\sin v_{m} \psi_{0}}{v_{m} \psi_{0}} \frac{e^{-i v_{m}(2 \pi-\varphi)}}{t_{m}} \\
& \psi_{1}^{+}=+i\left(\frac{V_{0} \epsilon}{k a}\right)\left(\frac{k a}{2}\right)^{2 / 3} \sum_{m=1}^{\infty} \frac{\sin v_{m} \psi_{0}}{v_{m} \psi_{0}} \frac{e^{-i v_{m}}}{t_{m}}
\end{aligned}
$$

where

$$
\begin{gathered}
t_{m}=\frac{3 x_{m}^{2 / 3}}{2} e^{-\frac{i \pi}{3}} \\
v_{m}=k a+\left(\frac{k a}{2}\right)^{1 / 3} t_{m}
\end{gathered}
$$

and $x_{m}$ represents the $m$ th root of

$$
J_{2 / 3}(x)-J_{-2 / 3}(x)=0
$$


It is apparent that the expression in (35a) represents the waves traveling in the negative $\varphi$ direction and in $(35 \mathrm{~b})$, those traveling in the positive $\varphi$ direction.

Therefore, for large cylinders, the total magnetic field is

$$
H_{Z}=+\frac{V_{0} k}{\omega \mu a}\left(\frac{k a}{2}\right)^{2 / 3} \sum_{m=1}^{\infty} \frac{\sin v_{m} \psi_{0}}{v_{m} \psi_{0}}\left[\frac{e^{-i v_{m}^{(2 \pi-\varphi)}}+e^{-i v_{m} \varphi}}{t_{m}}\right]
$$

For sufficiently large values of $\varphi$ the serıes is rapidly convergent. For instance, with $k a=25$, the $m=4$ term is less than 1 percent of the $m=3$ term for $\varphi=20^{\circ}$.

To evaluate the integral in (27) for $\varphi$ small, we again consider the traveling waves to be highly attenuated at the end of a 360 degree path. Therefore, we can again substitute

$$
2 i e^{-i v \pi} \text { for } \frac{1}{\sin v \pi}
$$

in eq (27) and consider the integral along the lower half of the contour. This gives the expression for the traveling wave along the positive $\varphi$ direction. The expression now becomes

$$
\psi_{1}^{+}=+\frac{V_{0} \epsilon}{2 \pi k a} \int_{-i \epsilon-\infty}^{-i \epsilon+\infty} \frac{\sin v \psi_{0}}{v \psi_{0}} e^{-i v \varphi} \frac{H_{v}^{(2)}(k a)}{H_{v}^{(2 \prime}(k a)} d v .
$$

We evaluate the ratio of the Hankel functions above by using again the first term of the asymptotic expansion discussed in appendix II. We have from (II-11) and (II-12)

$$
\begin{aligned}
& H_{v}^{(2)}(k a) \cong-\frac{1}{i \sqrt{\pi}}\left(\frac{2}{k a}\right)^{1 / 3} \omega(t) \\
& H_{v}^{(2)^{\prime}}(k a) \cong \frac{1}{i \sqrt{\pi}}\left(\frac{2}{k a}\right)^{2 / 3} \omega^{\prime}(t)
\end{aligned}
$$

which gives for $v=k a+\left(\frac{k a}{2}\right)^{1 / 3} t$

$$
\frac{H_{v}^{(2)}(k a)}{H_{v}^{(2) \prime}(k a)} \cong-\left(\frac{k a}{2}\right)^{1 / 3} \frac{\omega(t)}{\omega^{\prime}(t)}
$$

This approximation is based on the assumption that $t$ remains finite as $(k a)$ increases. The integral in (37) implies an integration for $0<|t|<\infty$ and the approximation in (38) is valid for finite values of $t$. This apparent contradiction is compatible due to the exponentially decaying nature of the integrand in (37) and Watson's Lemma. Therefore, the major contribution to the integral is due to the finite values of $t$. Thus, taking the approximation of (38) and the first term of the expansion

$$
\begin{aligned}
& \frac{\sin v_{m} \psi_{0}}{v_{m} \psi_{0}}=\frac{\sin k a \psi_{0}}{k a \psi_{0}}\left[1-\frac{1}{2}\left(\frac{2}{k a}\right)^{2 / 3} t\left(1-k a \psi_{0} \cot k a \psi_{0}\right)\right. \\
& \\
& \left.\quad+\frac{1}{4}\left(\frac{2}{k a}\right)^{4 / 3} t^{2}\left(1-k a \psi_{0} \cot k a \psi_{0}+\frac{\left(k a \psi_{0}\right)^{2}}{2}\right)+\ldots\right]
\end{aligned}
$$

The integral in (37) with the new variable of integration, $t$, becomes

$$
\psi_{1}^{+}=-\frac{V_{0} \epsilon}{2 \pi k a} \frac{\sin k a \psi_{0}}{k a \psi_{0}}\left(\frac{k a}{2}\right)^{2 / 3} e^{i k a \varphi} \int_{-i \epsilon^{\prime}-\infty}^{-i \epsilon^{\prime}+\infty} e^{-i\left(\frac{k a}{2}\right)^{1 / 3} \varphi t} \frac{\omega(t)}{\omega^{\prime}(t)} d t .
$$

We now make the following change of variables:

$x=k a \varphi=2 \pi$ times the distance in wavelength from the center of the slot $2 x_{0}=2 k a \psi_{0}=2 \pi$ times the slot width in wavelength 


$$
\begin{aligned}
& c=2\left(\frac{k a}{2}\right)^{2 / 3}=\text { a curvature term } \\
& s=-i t=\text { change of variable of integration. }
\end{aligned}
$$

The expression in (40) now becomes

$$
\psi_{1}^{+}=\frac{\epsilon V_{0}}{\sqrt{2}} \frac{\sin x_{0}}{x_{0}} \frac{e^{-i x}}{\sqrt{x}} I
$$

where

$$
I=\sqrt{\frac{x}{c}} \frac{1}{2 \pi i} \int_{-\epsilon^{\prime}-i \infty}^{-\epsilon^{\prime}+i_{\infty}} e^{\frac{x}{c}} \frac{\omega(i s)}{\omega^{\prime}(i s)} d s
$$

The singularities of the integrand above lie on the left hand side of the line of integration in the $s$-plane. Since the origin is not a singularity we can express (42) as a Bromwhich integral with $\tau=x / c$

$$
I(\tau)=\sqrt{\tau} \frac{1}{2 \pi i} \int_{B r_{1}} e^{\tau s} \frac{\omega(i s)}{\omega^{\prime}(i s)} d s
$$

Therefore, with $\mathscr{S}$ representing the Laplace operator

$$
I(\tau)=\sqrt{\tau} \mathscr{L}^{-1} \frac{\omega(i s)}{\omega^{\prime}(i s)} .
$$

We next attempt to find an expansion of

$$
F(s)=\frac{\omega(i s)}{\omega^{\prime}(i s)}
$$

in inverse powers of $s$ to obtain an expansion of $I(\tau)$ for $\tau$ small. We have

Therefore

$$
\begin{aligned}
\omega(t) & =\sqrt{\frac{\pi}{3}} e^{-i \frac{2 \pi}{3}}(-t)^{1 / 2} H_{1 / 3}^{(2)}\left[\frac{2}{3}(-t)^{3 / 2}\right] \\
\omega^{\prime}(t) & =\sqrt{\frac{\pi}{3}} e^{-i \frac{\pi}{3}}(-t) H_{2 / 3}^{(2)}\left[\frac{2}{3}(-t)^{3 / 2}\right] .
\end{aligned}
$$

$$
F(s)=\frac{e^{-i \frac{\pi}{3}}}{\sqrt{-i s}} \frac{H_{1 / 3}^{(2)}\left[\frac{2}{3}(-i s)^{3 / 2}\right]}{H_{2 / 3}^{(2)}\left[\frac{2}{3}(-i s)^{3 / 2}\right]} .
$$

In order to develop the Hankel functions in their asymptotic expansions, we need to assure that the phase of the argument lies in the range $-2 \pi$ to $\pi$. We note that in (43) $s$ will range in value from $+i \infty$ to $-i \infty$. Therefore, we use the identity

in (47) and obtain

$$
\frac{H_{1 / 3}^{(2)}\left(Z^{\prime}\right)}{H_{2 / 3}^{(2)}\left(Z^{\prime}\right)}=e^{i \pi} \frac{H_{1 / 3}^{(2)}\left(Z^{\prime} e^{-i 3 \pi}\right)}{H_{2 / 3}^{(2)}\left(Z^{\prime} e^{-i 3 \pi}\right)}
$$

$$
F(s)=\frac{e^{-\frac{i \pi}{12}} H_{1 / 3}^{(2)}\left[-\sqrt{i} \frac{2}{3} s^{3 / 2}\right]}{\sqrt{s} H_{2 / 3}^{(2)}\left[-\sqrt{i} \frac{2}{3} s^{3 / 2}\right]} .
$$

Using the asymptotic expansions of the Hankel function we obtain for their ratio 


$$
\frac{H_{1 / 3}^{(2)}(Z)}{H_{2 / 3}^{(2)}(Z)}=e^{-i \pi / 6}\left[1+\frac{i}{6 Z}-\frac{7}{72 Z^{2}}-i \frac{7}{72 Z^{3}}+\ldots\right] .
$$

If we now let $Z=-\sqrt{i} \frac{2}{3} s^{3 / 2}$ and substitute (50) in (44) we obtain

$$
I(\tau)=e^{-i \frac{\pi}{4}} \sqrt{\tau} \mathscr{L}^{-1}\left[\frac{1}{\sqrt{s}}-\frac{\sqrt{i}}{4 s^{2}}+\frac{7 i}{32 s^{7 / 2}}+\frac{21}{\sqrt{i} 64 s^{5}}+\ldots\right] .
$$

Then employing the basic relation

it follows that

$$
\frac{1}{s^{v}}=\int_{0}^{\infty} e^{-\tau s} \frac{\tau^{v-1}}{\Gamma(v)} d \tau=\mathscr{L}\left[\frac{\tau^{v-1}}{\Gamma(v)}\right]
$$

where

$$
I(\tau)=\frac{e^{-i \frac{\pi}{4}}}{\sqrt{\pi}}\left(1-\frac{1}{4} \sqrt{\pi i} \tau^{3 / 2}+\frac{7}{512} \sqrt{\frac{\pi}{i}} \tau^{9 / 2}-\ldots\right)
$$

$$
\tau=\frac{x}{c}=\frac{k a \varphi}{2\left(\frac{k a}{2}\right)^{2 / 3}}
$$

Substituting (53) in (41) and letting $y=\frac{(k a \varphi)^{3 / 2}}{k a}$, we finally have

$\psi_{1}^{+}=+i \frac{V_{0} \epsilon}{2} \frac{\sin x_{0}}{x_{0}} \sqrt{\frac{2}{\pi x}} e^{-i(x-\pi / 4)}\left[\left(1-\frac{\sqrt{\pi}}{8} y+\frac{7 \sqrt{\pi}}{2048} y^{3}+\ldots\right)-i\left(\frac{\sqrt{\pi}}{8} y-\frac{7}{120} y^{2}+\frac{7 \sqrt{\pi}}{2048} y^{3}+\ldots\right)\right]$.

The curvature term, the series appearing in (54), is rapidly convergent for small $y$. It approaches unity as $(k a)$ approaches infinity. Therefore, the expression

$$
\psi_{10}^{+}=\left[+i \frac{V_{0} \epsilon}{2} \frac{\sin x_{0}}{x_{0}}\right] \sqrt{\frac{2}{\pi x}} e^{-i(x-\pi / 4)}
$$

which appears in (54) as a multiplying factor to the curvature term, should represent the field due to the slot on a flat sheet. However, taking the same limit of the original expression, eq (37), yields the following results:

$$
\lim _{k a \rightarrow \infty} \psi_{1}^{+}=\lim _{k a \rightarrow \infty}\left(+\frac{i V_{0} \epsilon}{2}\right) \frac{1}{2 x_{0}} \int_{-x_{0}}^{x_{0}} d x^{\prime} \frac{1}{\pi i} \int_{-i \epsilon^{\prime}-\infty}^{-i \epsilon+\infty} e^{-i v\left(\varphi-\varphi^{\prime}\right)} \frac{H_{v}^{(2)}(k a)}{k a H_{v}^{(2) \prime}(k a)} d v
$$

where $x_{0}=k a \psi_{0}$ and $x^{\prime}=k a \varphi^{\prime}$.

We have from Sensiper [1957] or Nishida [1960], with $x=k a_{\varphi}$

$$
\lim _{k a \rightarrow \infty} \frac{1}{\pi i} \int_{-i \epsilon-\infty}^{-i \epsilon+\infty} e^{-i v\left(\varphi-\varphi^{\prime}\right)} \frac{H_{v}^{(2)}(k a)}{k a H_{v}^{(2) \prime}(k a)} d v=H_{0}^{(2)}\left(x-x^{\prime}\right) .
$$

Therefore (56) becomes

$$
\psi_{10}^{+}=+\frac{i V_{0} \epsilon}{2} \frac{1}{2 x_{0}} \int_{-x_{0}}^{x_{0}} H_{0}^{(2)}\left(x-x^{\prime}\right) d x^{\prime} .
$$

The expression in (57) is the exact scaler function for the fields of a slot on a flat sheet with a constant electric field $V_{0} k / 2 x_{0}$ across a width of $\left(2 x_{0} / k\right)$. For a sufficiently small $x_{0}$, (57) becomes

$$
\psi_{10}^{+} \simeq+\frac{i V_{0} \epsilon}{2} H_{0}^{(2)}(x)
$$


and for a sufficiently large $x$

$$
\psi_{10}^{+} \simeq+\frac{i V_{0} \epsilon}{2} \frac{\sin x_{0}}{x_{0}} \sqrt{\frac{2}{\pi x}} e^{-i(x-\pi / 4)} .
$$

Since (59) is the first term of the asymptotic expansion of (57) and (59) is also equal to $(55)$, it is reasonable to assume that a more exact expression for (55) would be (57). Therefore, we can say that if $U_{0}$ is the expression for a slot on a flat conducting sheet, then the expression for a slot on a cylinder of large $(k a)$ and values of $\varphi$ small is

$$
\psi_{1}^{+}=U_{0}\left[\left(1-\frac{\sqrt{\pi}}{8} y+\frac{7 \sqrt{\pi}}{2048} y^{3}+\ldots\right)-i\left(\frac{\sqrt{\pi}}{8} y-\frac{7}{120} y^{2}+\frac{7 \sqrt{\pi}}{2048} y^{3}+\ldots\right)\right]
$$

where

$$
y=\frac{(k a \varphi)^{3 / 2}}{k a}
$$

A plot of the bracketed term of (60) is shown in figure 3.7. This result corresponds exactly with the conclusions of J. R. Wait in his analysis of currents induced on large spheres [Wait, 1956].

For a very narrow slot the expression of $U_{0}$ should be, from (58),

$$
U_{0}=+i \frac{V_{0} \epsilon}{2} H_{0}^{(2)}(x)
$$

where $x$ is the distance from the slot. Our derivation for the corresponding term, shown in (55), has only the first term of the asymptotic expansion of the Hankel function appearing in (61). Therefore, for narrow slots (61) may be used in conjunction with (60).

In conclusion we have, for large radius cylinders $(k a \geq 10)$, the expression for the magnetic field on the surface of the cylinder

$$
H_{Z}=\frac{i k^{2}}{\omega \mu \epsilon}\left[\psi_{1}^{+}+\psi_{1}^{-}\right]
$$

where $\psi_{1}^{+}$and $\psi_{1}^{-}$are the traveling wave components in the positive and negative $\varphi$ direction. The expressions for $\psi_{1}^{+}$, for a slot located at $\varphi=0^{\circ}$, has been discussed in detail in this section. From (35) we have

$$
\psi_{1}^{-}(\varphi)=\psi_{1}^{+}(2 \pi-\varphi)
$$

which indicates the symmetric distribution of the magnetic field about the slot.

Figures 3.8 and 3.9 show the plots of the ratio of the traveling wave component of the magnetic field due to a narrow slot on the cylinder to the field of a slot on a flat sheet. The computations are based on the approximations expressed in (35) and (54).

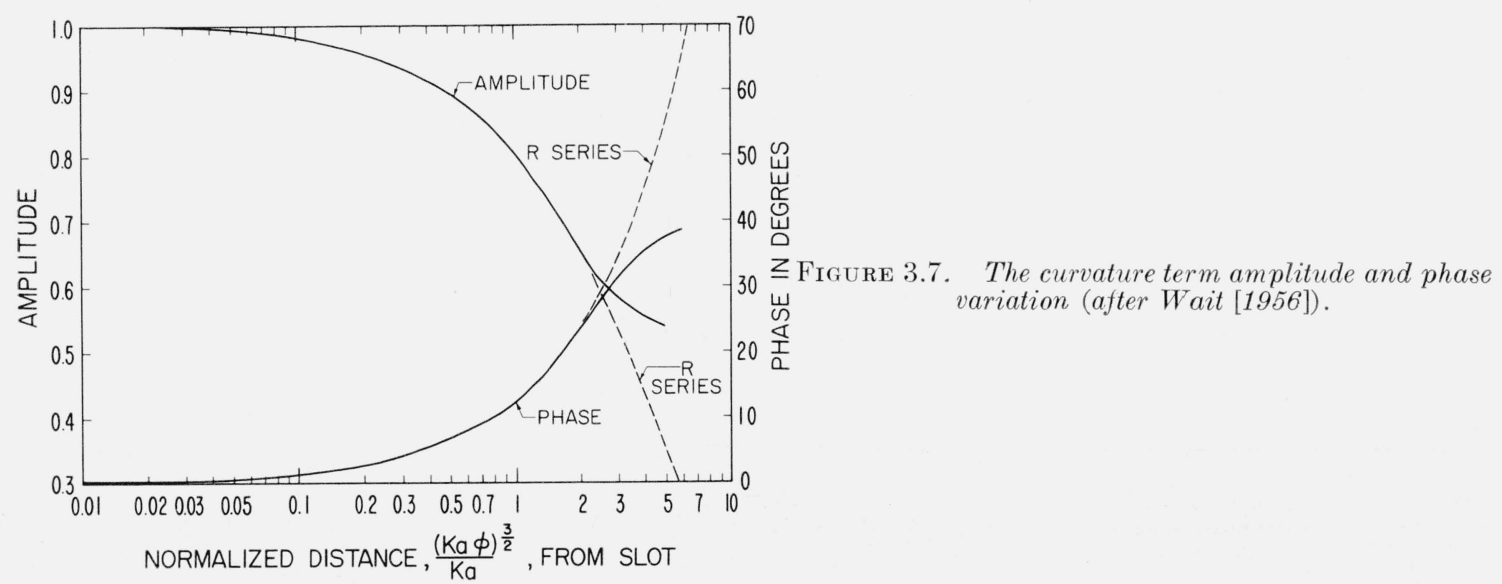




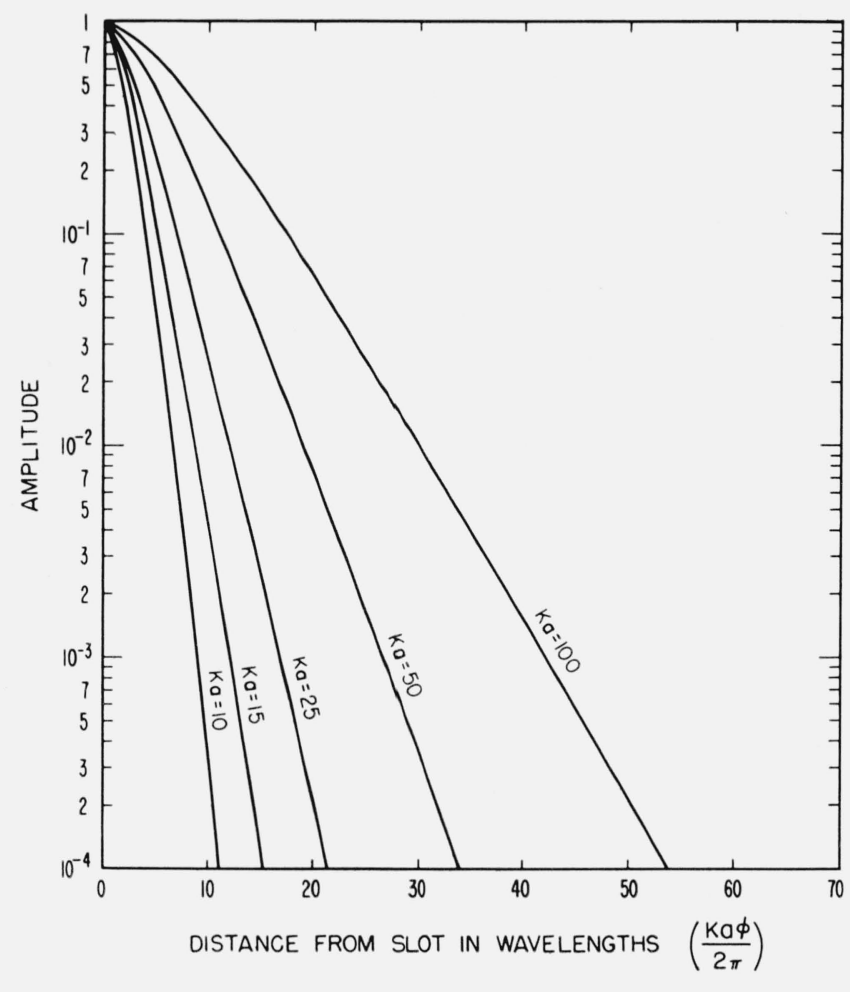

FIGURE 3.8. Magnitude of the ratio of the traveling wave current on the cylinder to that of a flat surface.

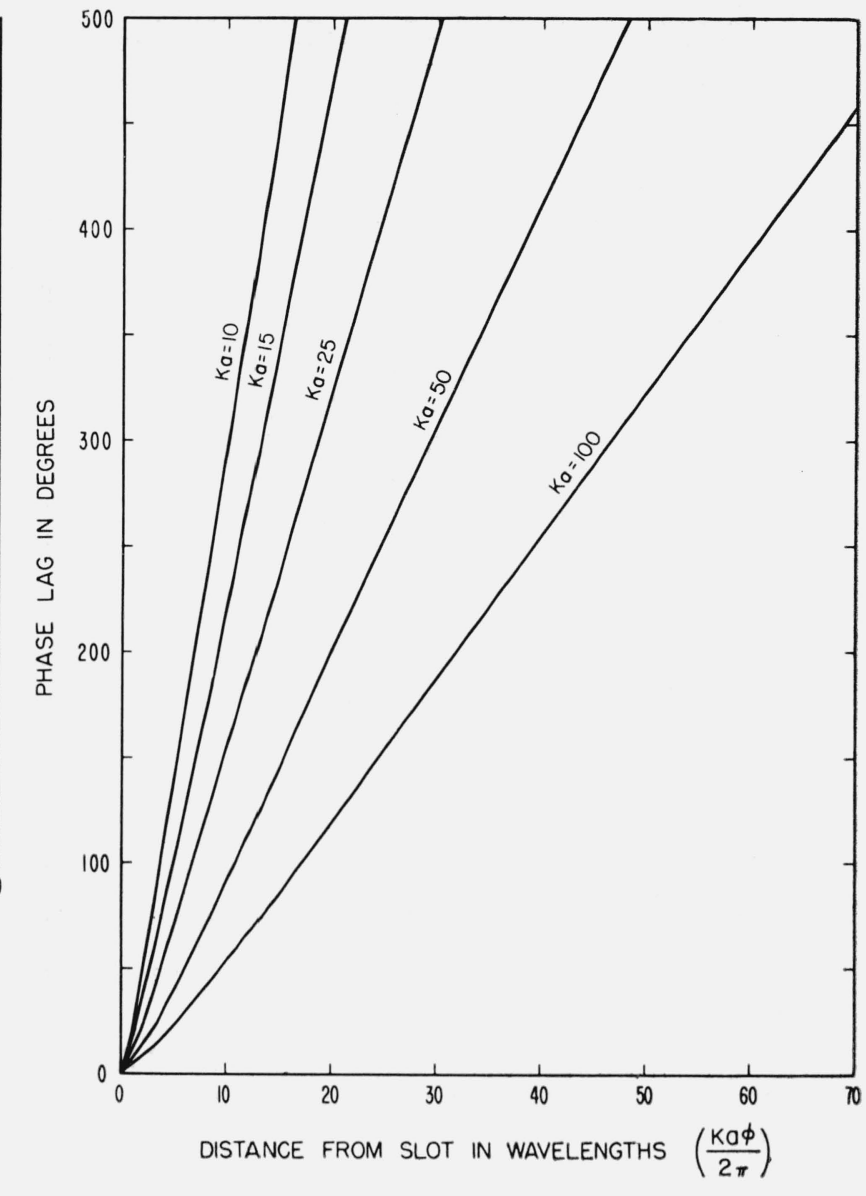

Figure 3.9. Phase of the ratio of the traveling wave current on the cylinder to that of a flat surface.

\subsection{Comparison of the Harmonic Series and Residue Series Evaluations}

In the evaluation of the surface fields by the harmonic series, we were able to show the order of the errors involved in the final evaluation. However, in the residue series evaluation, we did not attempt to indicate the order of the errors involved in the approximations. However, it is quite obvious that as $k a$ increases the residue series evaluation becomes increasingly accurate. On the other hand, as $k a$ increases, the use of the harmonic series becomes considerably more difficult. Since there is a range of values of $k a$, where both methods can be employed, we devote this section for a comparison of the results of the two methods. We perform this comparison by numerical computations for cylinder sizes with $k a=10,15$, and 25 .

Figure 3.10 shows the results of three computations. The solid curve is a plot of the magnitude of the magnetic field computed by the harmonic series (see sec. 3.2). According to the estimate of the remainder (see eq $(24)$ ) the order of the error by this method is $(1 / M)$ for small $\varphi$ and

$$
\frac{1}{M^{2} \varphi}\left[1+\left(\frac{k a}{M}\right)^{2}+M\left(\frac{k a}{M}\right)^{4}\right]
$$

for large $M \varphi$. Therefore, the errors in the computations of the solid curves of figure 3.10 are of order less than 1 percent over all values of $\varphi$. The points, in the figure, designated by the symbols $\odot$ and $\Delta$ were computed by the residue series method. For the set of points $\odot$, the computations were based on the first order approximation of the roots, $v_{m 2}$, of the Hankel 


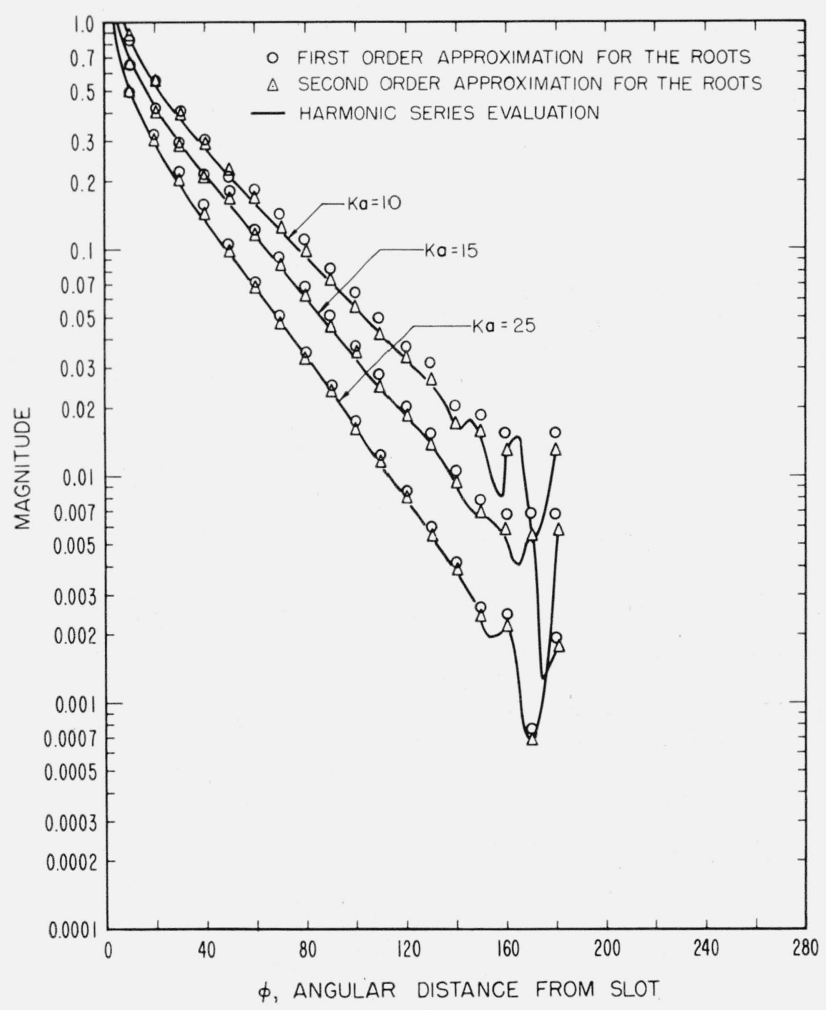

FiguRE 3.10. Comparison of residue series and harmonic series evaluation.

functions discussed in appendix II. On the other hand, the set of points, $\Delta$, were computed by using a second order approximation for the roots $v_{m 2}$. It is quite apparent from the figure that the second set of points are closer to the harmonic series evaluation, as expected. Actual comparison of the numerical values shows that the latter set is off by less than 2 percent of the harmonic series evaluation. A comparison of the phase of the field by the two methods, which is not shown, has a maximum deviation of 5 degrees for either set of roots.

It should be noted that, in the figure, the region very close to the slot was omitted for convenience. The agreement between the two methods of evaluation holds for points up to several slot widths. However, if the field distribution across the slot and its edge is desired, the harmonic series evaluation will yield a more accurate result for values of $k a<25$. The expression in (60) can be used for $k a>10$ provided the expression for a slot on a flat infinite sheet is known.

\section{Circumferential Slot Excitation}

In this section we will consider the case of a uniformly excited circumferential slot running entirely around the cylinder.

Describing the field distribution on the surface of the cylinder we have, with $V$ representing the voltage across the slot,

From (2-10) we have

$$
\begin{aligned}
& E_{Z}(r=a)=V \delta(Z) \\
& E_{\varphi}(r=a)=0 .
\end{aligned}
$$

$$
B_{n 2}(\zeta)=\frac{1}{2 \pi} \int_{Z_{1}}^{Z_{2}} d Z \int_{\varphi_{1}^{\prime}}^{\varphi_{2}^{\prime}} g_{1}(\varphi) g_{2}(Z) e^{i(n \varphi-\zeta Z)} d \varphi
$$

Using (1) in the expression above, we have

$$
B_{n 2}(\zeta)=\frac{1}{2 \pi} \int \delta(Z) e^{-i \xi Z} d Z \int_{-\pi}^{\pi} V e^{i n \varphi} d \varphi=V \frac{\sin n \pi}{n \pi}=\left\{\begin{array}{l}
1, n=0 \\
0, n \neq 0
\end{array}\right.
$$


Substitution of (2) into (2-13) and (2-14) leads to

and

$$
\psi_{1 t}=0
$$

$$
\psi_{2 t}=\frac{i \omega \mu \epsilon V}{2 \pi} \int_{c_{1}} \frac{e^{i \zeta Z} H_{0}^{(2)}\left(r \sqrt{k^{2}-\zeta^{2}}\right) d \zeta}{\left(k^{2}-\zeta^{2}\right) H_{0}^{(2)}\left(a \sqrt{k^{2}-\zeta^{2}}\right)}
$$

where $c_{1}$ is the contour defined in figure 2.2 .

Using (3), the equations in (2-8), and solving for the magnetic field on the surface of the cylinder, we have

and

$$
H_{Z}(r=a)=0
$$

$$
H_{\varphi}(r=a)=\frac{\omega \epsilon V a}{2 \pi i} \int_{c_{1}} \frac{e^{i \zeta Z} H_{0}^{(2) \prime}\left(a \sqrt{k^{2}-\zeta^{2}}\right) d \zeta}{a \sqrt{k^{2}-\zeta^{2}} H_{0}^{(2)}\left(a \sqrt{k^{2}-\zeta^{2}}\right)} .
$$

The problem, therefore, reduces to the evaluation of the integral

$$
I(Z)=\frac{1}{2 \pi i} \int_{c_{1}} \frac{e^{i \zeta Z} H_{0}^{(2)}\left(a \sqrt{k^{2}-\zeta^{2}}\right)}{a \sqrt{k^{2}-\zeta^{2}} H_{0}^{(2)}\left(a \sqrt{k^{2}-\zeta^{2}}\right)} d \zeta .
$$

For $Z>0$, we can deform the contour $c_{1}$ about the branch cut as shown in figure 4.1. The contours $c_{1}$ and $c_{2}$ are equivalent since for $R_{1} \rightarrow \infty$ the integrand converges to zero and the integral about the outer circle does not contribute to the value of $I$. We will next show that we can obtain an asymptotic expansion of (5) for $k Z<(2 k a)^{2}$.

The argument of the Hankel functions in (5) varies between $\pi / 4$ to $-\pi$ along the contour $c_{2}$. Therefore, with a sufficiently large $R_{0}$, we can take the asymptotic expansion of the ratio of the Hankel functions appearing in (5). With $y=a \sqrt{k^{2}-\zeta^{2}}$, we have

$$
\frac{H_{0}^{(2)}(y)}{H_{0}^{(2)}(y)}=-i\left\{1+\frac{1}{2 i y}-\frac{1}{2} \frac{1}{(2 i y)^{2}}+\frac{1}{(2 i y)^{3}}-\frac{25}{8} \frac{1}{(2 i y)^{4}}+0\left(y^{-5}\right)\right\} .
$$

Substitution of (6) in the integrand of (5) leads to

$$
I(k Z) \cong-\frac{1}{2 \pi i} \int_{c_{2}} e^{i \zeta k Z} d \zeta\left[\left(\frac{1}{2 y^{2}}-\frac{1}{8 y^{4}}\right)+i\left(\frac{1}{y}+\frac{1}{8 y^{3}}-\frac{25}{128 y^{5}}\right)+0\left(y^{-6}\right)\right]
$$

Therefore, in general, the asymptotic expansion of $I(k Z)$ will have the form

$$
\begin{gathered}
I(k Z)=-\sum_{n=0}^{m-1} a_{n} g_{n}(k Z)+i b_{n} f_{n}(k Z)+R_{m} \\
R_{m}=0\left[(k a)^{-2 m} \int_{c_{2}} \frac{\epsilon^{i \zeta k Z}}{\left(1-\zeta^{2}\right)^{m}} d \zeta\right] \\
g_{n}(k Z)=\frac{1}{2 \pi i} \int_{c_{2}} \frac{e^{i \xi k Z}}{\left(1-\zeta^{2}\right)^{n}} d \zeta \\
f_{n}(k Z)=\frac{1}{2 \pi i} \int_{c_{2}} \frac{e^{i \zeta k Z}}{\left(1-\zeta^{2}\right)^{n+1 / 2}}
\end{gathered}
$$

and the first few coefficients $a_{n}$ and $b_{n}$ are:

$$
a_{0}=0 \quad a_{1}=\frac{1}{2(k a)^{2}} \quad a_{2}=\frac{-1}{8(k a)^{4}}
$$




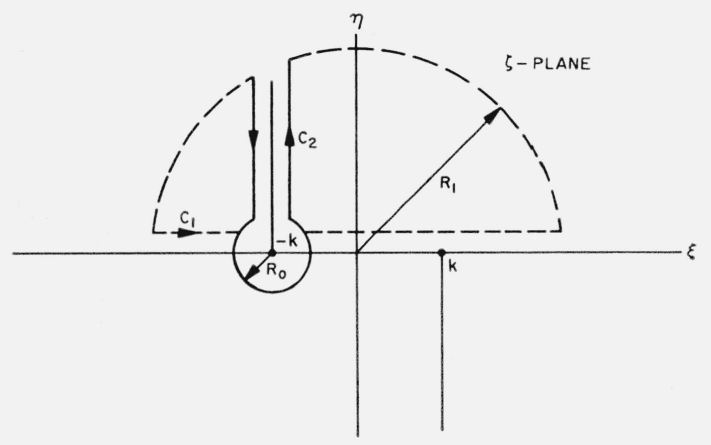

FiguRE 4.1. Equivalent contours for the integral $I(Z)$.

$$
b_{0}=\frac{1}{k a} \quad b_{1}=\frac{1}{8(k a)^{3}} \quad b_{2}=\frac{-25}{128} \frac{1}{(k a)^{5}} .
$$

From complex variable theory (10) becomes

$$
g_{n}(Z)=\left\{\text { Residue }\left[\frac{e^{i k Z \zeta}}{\left(1-\zeta^{2}\right)^{n}}\right]_{\zeta=-1}\right\}=\frac{1}{(n-1) !} \frac{d^{n-1}}{d \zeta^{n-1}}\left[\frac{e^{i k Z \zeta}}{(1-\zeta)^{n}}\right]_{\zeta=-1} .
$$

From Courant and Hilbert [1953, page 482], we have

$$
H_{\lambda}^{(2)}(k Z)=-\frac{1}{\pi i} \frac{\Gamma(1 / 2-\lambda)}{\Gamma(1 / 2)}\left(\frac{k Z}{2}\right)^{\lambda} \int_{c_{2}} e^{i k Z \zeta}\left(\zeta^{2}-1\right)^{\lambda-1 / 2} d \zeta
$$

Therefore, (11) becomes

$$
f_{n}(Z)=-\frac{1}{2} \frac{\Gamma\left(\frac{1}{2}\right)}{\Gamma\left(n+\frac{1}{2}\right)}\left(\frac{k Z}{2}\right)^{n} e^{\imath \frac{\pi}{2}(2 n+1)} H_{-n}^{(2)}(k Z),
$$

Using the well known identity

$$
H_{-n}^{(2)}(k Z)=e^{-i n \pi} H_{n}^{(2)}(k Z)
$$

we finally have for (11)

$$
f_{n}(Z)=-\frac{i}{2} \frac{\Gamma\left(\frac{1}{2}\right)}{\Gamma\left(n+\frac{1}{2}\right)}\left(\frac{k Z}{2}\right)^{n} H_{n}^{(2)}(k Z),
$$

It now remains to determine (9), which is the order of magnitude of the remainder term in (8). For $0<k Z<1$,

and for $k Z>1$

$$
\int_{c_{2}} \frac{e^{i k Z_{\zeta}} d \zeta}{\left(1--\zeta^{2}\right)^{m}}=\left[0\left(\frac{1}{2^{2 m}}\right)\right]
$$

$$
\int_{c_{2}} \frac{e^{i k Z \xi} d \zeta}{\left(1-\zeta^{2}\right)^{m}}=0\left(\left[\frac{k Z}{2}\right]^{m-1}\right)
$$

Therefore

$$
R_{m}=0\left[\frac{1}{(2 k a)^{2 m}}\left(1+(k Z)^{m-1}\right)\right] .
$$

Therefore (5) can now be expressed in the following asymptotic form with $\frac{(k a)^{2}}{k Z}$ sufficiently large: 


$$
\begin{aligned}
I(Z) \cong-\frac{1}{2}\left[\frac{e^{-i k Z}}{2(k a)^{2}}-\frac{e^{-i k Z}(1+i k Z)}{16(k a)^{4}}\right. & +\frac{1}{k a} H_{0}^{(2)}(k Z) \\
& \left.+\frac{1}{8} \frac{k Z}{(k a)^{3}} H_{1}^{(2)}(k Z)-\frac{25}{384} \frac{(k Z)^{2}}{(k a)^{5}} H_{2}^{(2)}(k Z)\right]+0\left[\frac{1+(k Z)^{2}}{(2 k a)^{6}}\right] .
\end{aligned}
$$

Therefore the magnetic field on the surface of the cylinder excited by a circumferential slot is, for $\frac{k Z}{(2 k a)^{2}}<<1$

$$
H_{\varphi}(Z) \cong-\frac{\omega \epsilon V}{2} H_{0}^{(2)}(k Z)\left[1+\left(\frac{1}{2 k a}-\frac{1+i k Z}{2(2 k a)^{3}}\right) \frac{e^{-i k Z}}{H_{0}^{(2)}(k Z)}+\frac{1}{2} \frac{k Z}{(2 k a)^{2}} \frac{H_{1}^{(2)}(k Z)}{H_{0}^{(2)}(k Z)}-\frac{25}{24} \frac{(k Z)^{2}}{(2 k a)^{4}} \frac{H_{2}^{(2)}(k Z)}{H_{0}^{(2)}(k Z)}\right]
$$

It is quite obvious that the bracketed term in (20) approaches unity as $(k a)$ approaches infinity. As we let $(k a)$ approach infinity, the problem reduces to the case of an infinite narrow slot, which is also the limiting case of $(20)$.

Figures 4.2 and 4.3 are plots of the magnitude and phase of

$$
f(Z)=1+\frac{1}{2 k a}\left(1-\frac{1+i k Z}{2(2 k a)^{2}}\right) \frac{e^{-i k Z}}{H_{0}^{(2)}(k Z)}+\frac{1}{2} \frac{k Z}{(2 k a)^{2}} \frac{H_{1}^{(2)}(k Z)}{H_{0}^{(2)}(k Z)}-\frac{25(k Z)^{2}}{24(2 k a)^{4}} \frac{H_{2}^{(2)}(k Z)}{H_{0}^{(2)}(k Z)}
$$

where various values of $(k a)$ were used as parameters and plotted as a function of normalized axial distances, $(Z / 2 a)$. It can be seen from the curves that there is an increase in magnitude of $f(Z)$ which shows the contribution of the various sections of the source whose path of propagation is along a spiral path on the surface.

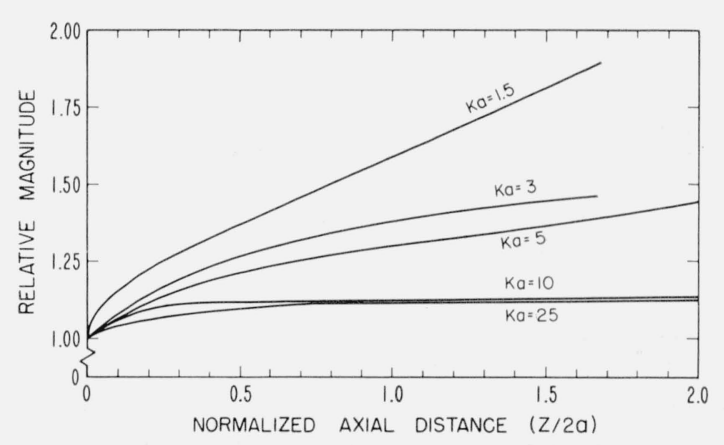

Figure 4.2. Ratio of the magnetic field on the cylinder to $\mathrm{H}_{0}{ }^{(2)}(\mathrm{kZ})$.

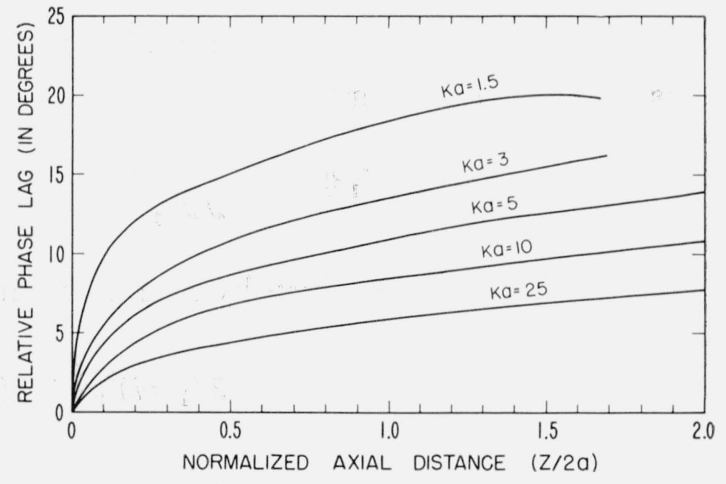

FIgure 4.3. The phase of the ratio of the magnetic field on the cylinder to $\mathrm{H}_{0}^{(2)}(\mathrm{kZ})$.

\section{Mutual Admittance of Slots Coupled Externally}

In this analysis we wish to express the Mutual Admittance of a pair of slots coupled to each other by their External Fields. By "External Fields" we mean the electromagnetic field region excluding the transmission line regions feeding the slots. To obtain the expression for the "External Mutual Admittance" we shall assume that the pair of slots are fed by independent transmission lines and that the slots are shunt elements to their respective feed lines.

The external magnetic field of a single slot can be expressed as (see Oliner [1957])

$$
H_{e}(r)=\iint_{S}(\vec{n} \times \vec{E}) \cdot y_{h} d S
$$


where $E$ is the electric field across the slot, $y_{h}$ is the dyadic Green's function for the exterior region of the slot and $S$ is the aperture of the slot cut in a conducting surface. Therefore, the external field due to a pair of slots can be expressed as

$$
\left.H_{e}(r)=\iint_{S_{1}}\left(\vec{n} \times \vec{E}_{1}\right) \cdot y_{h} d S+\iint_{S_{2}} \overrightarrow{(n} \times E_{2}\right) y_{h} d S
$$

where $E_{1}$ and $E_{2}$ represent the respective electric fields across the slots, $S_{1}$ and $S_{2}$ their respective apertures.

The fields of the slots in their transmission lines can be expressed in terms of voltages and currents. According to Marcuvitz and Schwinger [1951], the internal magnetic field due to a slot in a waveguide is expressed as

$$
H_{i}(r)=\frac{1}{2}\left(I^{(1)}+I^{(2)}\right) \beta^{(1)}(r)-i \frac{Y_{0}}{2}\left(V^{(1)}+V^{(2)}\right) \beta^{(2)}(r)-i \iint_{S}(\vec{n} \times \vec{E}) \cdot \beta d S
$$

where $\beta$ is the Green's Function Diadic for the waveguide and $\beta^{(1)}, \beta^{(2)}$ are the standing wave type mode functions for the waveguide.

The continuity of the tangential field required that $H_{i}(r)=H_{e}(r)$ at the slot. Therefore, using (2) and (3) for slot (1) we have

$$
\iint_{S_{1}}\left(\vec{n} \times \vec{E}_{1}\right) \cdot\left(y_{h}+i \beta\right) d S+\iint_{S_{2}}\left(\vec{n} \times \vec{E}_{2}\right) \cdot y_{h} d S=\frac{1}{2}\left(I_{1}^{(1)}+I_{1}^{(2)}\right) \beta_{1}^{(1)}(r)-i \frac{Y_{0}}{2}\left(V_{1}^{(1)}+V_{1}^{(2)}\right) \beta_{1}^{(2)}(r) .
$$

If this slot and its external load can be represented as a shunt element in the transmission line then

$$
I_{1}=I_{1}^{(1)}=-I_{1}^{(2)} \text { and } V_{1}=V_{1}^{(1)}=V_{1}^{(2)}
$$

thus reducing eq (4) into

$$
-i Y_{0} V_{1} \beta_{1}^{(2)}(r)=\iint_{S_{1}}\left(\vec{n} \times \vec{E}_{1}\right) \cdot\left(y_{h}+i \beta_{1}\right) d S+\iint_{S_{2}}\left(\vec{n} \times \vec{E}_{2}\right) \cdot y_{h} d S .
$$

But, by the definitions given by Marcuvitz and Schwinger [1951]

$$
2 I_{1}=i Y_{0} \iint_{S_{1}}\left(\vec{n} \times \vec{E}_{1}\right) \cdot \beta_{1}^{(2)} d S=I_{1}^{\prime}
$$

where $I_{1}^{\prime}$ is the total current in the shunt admittance. Multiplying (5) by $(\vec{n} \times \vec{E})$ and integrating over $S$, we obtain

$$
\begin{aligned}
-V_{1} I_{1}^{\prime}=\iint_{S_{1}}\left[\iint_{S_{1}}\left(\vec{n} \times \vec{E}_{1}\right) \cdot\left(y_{h}+i \beta_{1}\right) d S\right] \cdot\left(\vec{n} \times \vec{E}_{1}\right) d S & \\
& \quad+\iint_{S_{1}}\left[\iint_{S_{2}}\left(\vec{n} \times \vec{E}_{2}\right) \cdot y_{h} d S\right] \cdot\left(\vec{n} \times \vec{E}_{1}\right) d S .
\end{aligned}
$$

We can write a similar expression for the second slot in terms of its characteristic equations, thus

$$
\begin{aligned}
-V_{2} I_{2}^{\prime}=\iint_{S_{2}}\left[\iint_{S_{2}}\left(\vec{n} \times \vec{E}_{2}\right) \cdot\left(y_{h}+i \beta_{2}\right) d S\right] \cdot\left(\vec{n} \times \vec{E}_{2}\right) d S & \\
& +\iint_{S_{2}}\left[\iint_{S_{1}}\left(\vec{n} \times \vec{E}_{1}\right) \cdot y_{h} d S\right] \cdot\left(\vec{n} \times \vec{E}_{2}\right) d S .
\end{aligned}
$$


Figure 5.1 represents an equivalent circuit whose elements we shall identify in terms of the expressions discussed above. Writing the network equations for the equivalent circuit we find

$$
\begin{aligned}
& I_{1 s}=Y_{11}^{\prime} V_{1 s}+Y_{12} V_{2 s} \\
& I_{2 s}=Y_{12} V_{1 s}+Y_{22}^{\prime} V_{2 s} .
\end{aligned}
$$

Multiplying the above equations by $V_{1 s}$ and $V_{2 s}$ respectively and considering ideal transformers (1) and (2) we have

$$
\begin{aligned}
& V_{1} I_{1}^{\prime}=V_{1 s} I_{1 s}=Y_{11}^{\prime} V_{1 s}^{2}+Y_{12} V_{2 s} V_{1 s} \\
& V_{2} I_{2}^{\prime}=V_{2 s} I_{2 s}=Y_{12} V_{1 s} V_{2 s}+Y_{22}^{\prime} V_{2 s}^{2} .
\end{aligned}
$$

Comparing equations (10a) and (10b) with (7) and (8) we immediately observe the equivalence, which leads to

$$
Y_{12}=-\frac{\iint_{S_{2}}\left[\iint_{S_{1}}\left(\vec{n} \times \vec{E}_{1}\right) \cdot y_{h} d S\right] \cdot \overrightarrow{\left(n \times \vec{E}_{2}\right) d S}}{V_{1 s} V_{2 s}}
$$

where $V_{1 s}$ and $V_{2 s}$ can be the slot voltages and the bracketed integral represents the magnetic field due to slot (1) across slot (2).

Considering the short circuit condition of the secondary $\left(V_{2}=0\right)$ and eqs (6), (7) and (10a) we obtain an expression for the self impedance of slot (1) at the transmission line terminals

$$
\frac{1}{Y_{11}}=\frac{N_{1}^{2}}{Y_{11}^{\prime}}=\frac{\iint_{S_{1}}\left[\iint_{S_{1}}\left(\vec{n} \times \vec{E}_{1}\right) \cdot\left(\mathscr{y}_{n}+i \beta_{1}\right) d S\right] \cdot\left(\vec{n} \times \vec{E}_{1}\right) d S}{Y_{01}^{2}\left[\iint_{S_{1}}\left(\vec{n} \times \vec{E}_{1}\right) \cdot \beta_{1}^{(2)} d S\right]^{2}} .
$$

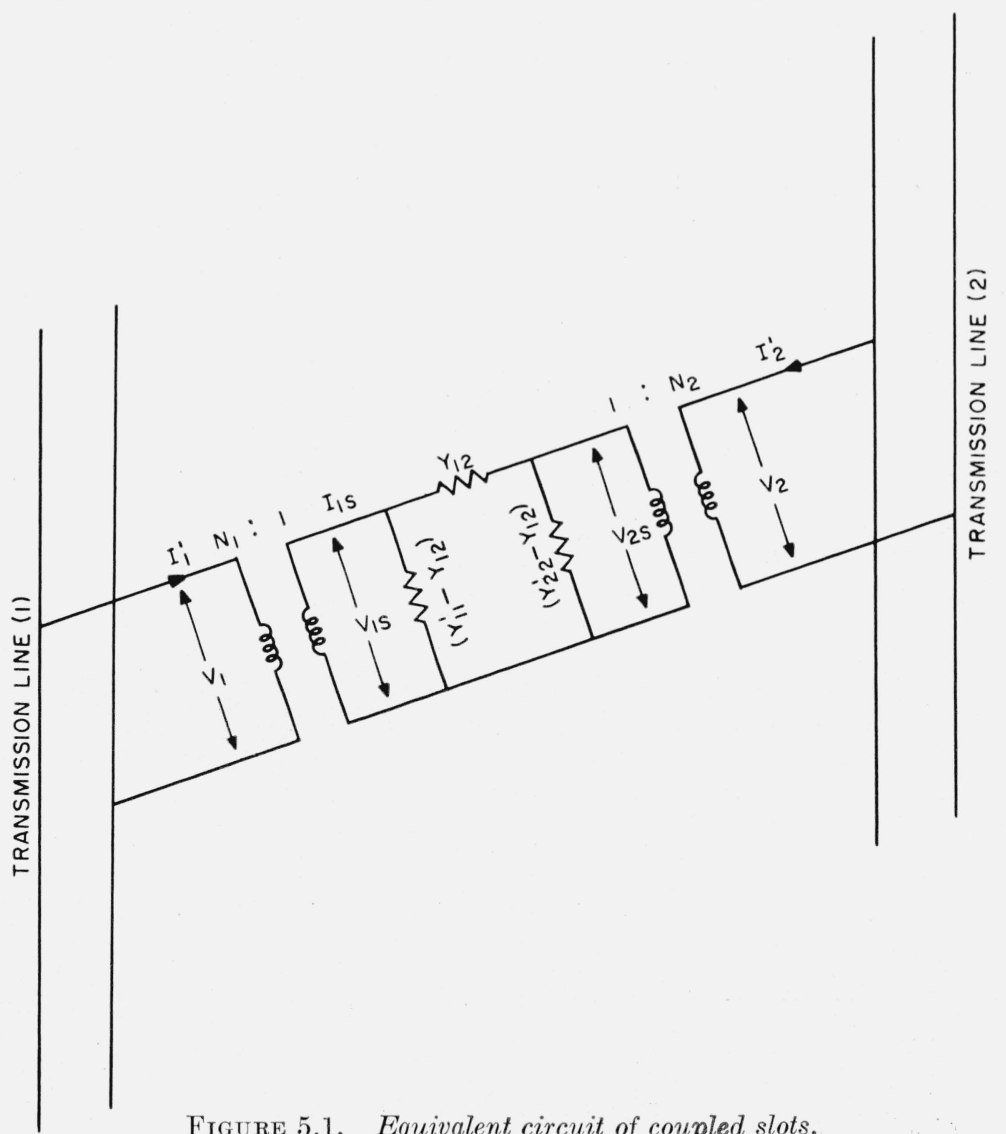

Figure 5.1. Equivalent circuit of coupled slots. 
Similarly the self impedance of the second slot is

$$
\frac{1}{Y_{22}}=\frac{N_{2}^{2}}{Y_{22}^{\prime}}=\frac{\left.\iint_{S_{2}}\left[\iint_{S_{2}}\left(\vec{n} \times \vec{E}_{2}\right) \cdot\left(\mathscr{y}_{n}+i \beta_{2}\right) d S\right] \cdot \overrightarrow{(n} \times \vec{E}_{2}\right) d S}{Y_{02}^{2}\left[\iint_{S_{2}}\left(\vec{n} \times \vec{E}_{2}\right) \cdot \beta_{2}^{(2)} d S\right]^{2}} .
$$

These are the same expressions as those obtained by Oliner in his study of impedance of narrow slots [Oliner, 1957]. It remains to show the expressions for $N_{1}$ and $N_{2}$. Considering the short circuit conditions again, we have

where,

$$
V_{1}=N_{1} V_{1 s} \text { or } N_{1}=\frac{I_{1}}{V_{1 s} Y_{11}}
$$

Therefore,

$$
I_{1}=i Y_{01} \iint_{S_{1}}\left(\vec{n} \times \vec{E}_{1}\right) \cdot \beta_{1}^{(2)} d S
$$

Similarly

$$
N_{1}=\frac{i Y_{01} \iint_{S_{1}}\left(\vec{n} \times \vec{E}_{1}\right) \cdot{ }^{\prime} \beta_{1}^{(2)} d S}{Y_{11} V_{1 s}} .
$$

$$
N_{2}=\frac{i Y_{02} \iint_{S_{2}}\left(\vec{n} \times \vec{E}_{2}\right) \cdot \beta_{2}^{(2)} \cdot d S}{Y_{22} V_{2 s}} .
$$

Physically, $N_{1}$ and $N_{2}$ represent the conversion factors of the slot voltages to the transmission line voltages of the waveguides. Such a number is necessary to evaluate the coupling coefficient, which can only be measured from one of the transmission lines feeding the coupled system.

\section{Appendix I. Evaluation of $S_{k}=\sum_{n=1}^{\infty} \frac{\sin n \psi_{0}}{n \psi_{0}} \frac{\cos n \varphi}{n^{k}}$}

In this appendix we evaluate the sum $S_{k}$ of the infinite series

$$
S_{k}=\sum_{n=1}^{\infty} \frac{\sin n \psi_{0}}{n \psi_{0}} \frac{\cos n \varphi}{n^{k}}
$$

for $k=1,2,4, \ldots$ With $k$ odd, the series is rather difficult to evaluate. Since we have avoided these cases in the text, with the exception of $k=1$, we shall discuss the series with $k$ even and then evaluate $S_{1}$ for values of $\psi_{0}$ small.

We can write (1) as the sum of two series as follows:

$$
S_{k}=\frac{1}{2 \psi_{0}}\left[\sum_{n=1}^{\infty} \frac{\sin n\left(\varphi+\psi_{0}\right)}{n^{k+1}}-\sum_{n=1}^{\infty} \frac{\sin n\left(\varphi-\psi_{0}\right)}{n^{k+1}}\right]
$$

We have from Jeffreys and Jeffreys [1956] for integer values of $r>1$

$$
\sum_{n=1}^{\infty} \frac{\sin 2 n \pi t}{n^{2 r-1}}=\frac{(2 \pi)^{2 r-1}(-1)^{r}}{2(2 r-1) !} \psi_{2 r-1}(t)
$$

where $\psi_{2 r-1}(t)$ are the Bernoulli polynomials and can be represented by the coefficients of $Z^{r} / r$ ! in the expansion

$$
\frac{Z\left(e^{Z t}-1\right)}{e^{Z}-1}=\sum_{r=0}^{\infty} \psi_{r}(t) \frac{Z^{r}}{r !}
$$


Substituting the expression in (3) into (2), we obtain for even $k \geq 2$

$$
S_{k}=\frac{1}{2 \psi_{0}} \frac{(2 \pi)^{k+1}(-1)^{2}}{2(k+1) !}\left[\psi_{k+1}\left(\frac{\varphi+\psi_{0}}{2 \pi}\right)-\psi_{k+1}\left(\frac{\varphi-\psi_{0}}{2 \pi}\right)\right] .
$$

We evaluate the specific case of $k=2$ and $k=4$. From (4) we have

$$
\begin{aligned}
& \psi_{3}(t)=t^{3}-\frac{3}{2} t^{2}+\frac{1}{2} t \\
& \psi_{5}(t)=t^{5}-\frac{5}{2} t^{4}+\frac{5}{3} t^{3}-\frac{1}{6} t .
\end{aligned}
$$

Substituting (6) into (5) and simplifying we finally obtain

$$
\begin{aligned}
& S_{2}=\frac{1}{12}\left[2 \pi^{2}-6 \pi \varphi+3 \varphi^{2}+\psi_{0}^{2}\right] \\
& S_{4}=\frac{\pi^{4}}{90}-\frac{\varphi^{4}}{48}+\frac{\pi}{12} \varphi^{3}-\left(\frac{\pi^{2}}{12}+\frac{\psi_{0}^{2}}{24}\right) \varphi^{2}+\frac{\pi}{12} \psi_{0}^{2} \varphi-\left(\frac{\pi^{2}}{36}+\frac{\psi_{0}^{2}}{240}\right) \psi_{0}^{2}
\end{aligned}
$$

We now proceed to evaluate $S_{1}$, for which one cannot employ the identity in (3). We have

$$
S_{1}=\frac{1}{\psi_{0}} \sum_{n=1}^{\infty} \frac{\sin n \psi_{0} \cos n \varphi}{n^{2}}=\frac{1}{2 \psi_{0}} \int^{\psi_{0}}\left[\sum_{n=1}^{\infty} \frac{\cos n\left(\varphi+\psi_{0}\right)}{n}+\sum_{n=1}^{\infty} \frac{\cos n\left(\varphi-\psi_{0}\right)}{n}\right] d \psi_{0} .
$$

Now consider the following function for $0 \leq \arg x \leq \pi$

$$
S(x)=\sum_{n=1}^{\infty} \frac{e^{i n x}}{n}=\int \frac{e^{i x} i d x}{\left(1-e^{i x}\right)}=-\ln \left(1-e^{i x}\right) .
$$

Then, for $x$ real and $-\pi \leq x \leq \pi$, we have

$$
\sum_{n=1}^{\infty} \frac{\cos n x}{n}=\operatorname{Re}[S(x)]=-\ln 2\left(\sin \frac{x}{2}\right) .
$$

Substituting (10) into (8), we obtain

$$
S_{1}=\frac{(-1)}{2 \psi_{0}} \int^{\psi_{0}}\left[\ln \left(2 \sin \frac{\varphi+\psi_{0}}{2}\right)+\ln \left(2 \sin \frac{\varphi-\psi_{0}}{2}\right)\right] d \psi_{0} .
$$

For $\psi_{0}$ small and $\psi_{0} \leq \varphi \leq \pi-\psi_{0}$, we have

$$
S_{1} \cong \frac{(-1)}{2 \psi_{0}} \int^{\psi_{0}}\left[\ln 2\left(\sin \frac{\varphi}{2}+\frac{\psi_{0}}{2} \cos \frac{\varphi}{2}\right)+\ln 2\left(\sin \frac{\varphi}{2}-\frac{\psi_{0}}{2} \cos \frac{\varphi}{2}\right)\right] d \psi_{0} .
$$

Carrying out the integration and simplifying we obtain

$$
S_{1}=1-\frac{1}{2} \ln 4\left[\sin ^{2} \frac{\varphi}{2}-\left(\frac{\psi_{0}}{2}\right)^{2} \cos ^{2} \frac{\varphi}{2}\right]+\frac{\tan \varphi / 2}{\psi_{0}} \ln \left[\frac{\tan (\varphi / 2)-\left(\psi_{0} / 2\right)}{\tan (\varphi / 2)+\left(\psi_{0} / 2\right)}\right]+\frac{1}{2 \psi_{0}} f_{1}(\varphi)
$$

where $f_{1}(\varphi)$ is the constant of integration. From (8) and (10) we have

$$
S_{1}\left(\varphi, \psi_{0}=0\right)=-\ln \left(2 \sin \frac{\varphi}{2}\right)
$$

From (13) we have

$$
\lim _{\psi_{0} \rightarrow 0} S_{1}\left(\varphi, \psi_{0}\right)=-\ln 2 \sin \frac{\varphi}{2}+\lim _{\psi_{0} \rightarrow 0} \frac{f_{1}(\varphi)}{2 \psi_{0}} .
$$

This will only be satisfied if $f_{1}(\varphi) \equiv 0$. In (13) the third member of the expression at first glance seems to be singular for $\psi_{0}=0$. However, the limit of this term as $\psi_{0} \rightarrow 0$ is unity. 
Therefore, (13) becomes

$$
S_{1} \cong 1+\left(\frac{\tan \varphi / 2}{\psi_{0}}-\frac{1}{2}\right) \ln \left(2 \sin \frac{\varphi}{2}-\psi_{0} \cos \frac{\varphi}{2}\right)-\left(\frac{\tan \varphi / 2}{\psi_{0}}+\frac{1}{2}\right) \ln \left(2 \sin \frac{\varphi}{2}+\psi_{0} \cos \frac{\varphi}{2}\right) .
$$

Special care is needed to evaluate $S_{1}$ at the point where $\psi_{0}=\varphi$ and $\varphi=\pi$. When we take the limit of $S_{1}$ as $\varphi$ approaches $\psi_{0}$ and $\pi$ we obtain

$$
\begin{aligned}
& S_{1}\left(\varphi=\psi_{0}\right)=1-\ln 2 \psi_{0} \\
& S_{1}(\varphi=\pi)=-\ln 2 .
\end{aligned}
$$

To evaluate $S_{1}$ for $0 \leq \varphi \leq \psi_{0}$, we need to reconsider the problem beginning with (8). Thus

$$
S_{1}=-\frac{1}{2 \psi_{0}} \int^{\varphi}\left[\sum_{n=1}^{\infty} \frac{\cos n\left(\varphi+\psi_{0}\right)}{n}-\sum_{n=1}^{\infty} \frac{\cos n\left(\varphi-\psi_{0}\right)}{n}\right] d \varphi
$$

Using (10) to evaluate the sums in (18) we take for $\varphi$ small and $0 \leq \varphi \leq \psi_{0}$

$$
S_{1} \cong-\frac{1}{2 \psi_{0}} \int^{\varphi}\left[\ln 2\left(\sin \frac{\psi_{0}}{2}+\frac{\varphi}{2} \cos \frac{\psi_{0}}{2}\right)-\ln 2\left(\sin \frac{\psi_{0}}{2}-\frac{\varphi}{2} \cos \frac{\psi_{0}}{2}\right)\right] d \varphi .
$$

Carrying out the integration and simplifying, we obtain

$$
S_{1} \cong-\frac{1}{2 \psi_{0}}\left[2 \tan \frac{\psi_{0}}{2} \ln 4\left(\tan ^{2} \frac{\psi_{0}}{2}-\left(\frac{\varphi}{2}\right)^{2}\right)-4 \tan \frac{\psi_{0}}{2}+\varphi \ln \frac{\tan \psi_{0} / 2+\varphi / 2}{\tan \psi_{0} / 2-\varphi / 2}\right] .
$$

As a check we note that at $\varphi=\psi_{0}(20)$ agrees with (17) if we assume $\psi_{0}$ small as in (17). An important point to obtain from $(20)$ is $S_{1}$ at $\varphi=0$. The last term in (20) approaches zero as $\varphi$ approaches zero. Therefore,

$$
S_{1}\left(\psi_{0}, \varphi=0\right)=\frac{2}{\psi_{0}} \tan \frac{\psi_{0}}{2}\left[1-\ln \left(2 \tan \frac{\psi_{0}}{2}\right)\right] .
$$

If $\psi_{0}$ is small (21) becomes

$$
S_{1}\left(\psi_{0}, \varphi=0\right)=1-\ln \psi_{0} .
$$

This completes the evaluation of $S_{1}$ for $\psi_{0}$ small for all values of $\varphi$.

\section{Appendix II. An Expansion of Hankel Functions in Terms of Airy Integrals}

In this appendix we will show an expansion of the Hankel Function of large complex argument in terms of the Airy integrals and its derivatives. This expansion is generally employed for real values of the argument of the Hankel function [Bremmer, 1949; Wait, 1959]. However, it is easy to show that the expansion is valid for the half-plane defined by

$$
-\left(\frac{\pi}{2}+\epsilon\right)<\arg Z<\frac{\pi}{2}-\epsilon
$$

where $Z$ is the argument of the Hankel function.

We consider the integral representation of the Hankel function

$$
H_{v}^{(2)}(Z)=-\frac{1}{\pi i} \int_{L 1} e^{-Z \sinh \zeta+v \zeta} d \zeta
$$

where the path of integration is as shown in figure II.1. To investigate the region of convergence we take

$$
Z=x+i y \text { and } \zeta=\xi+i \eta \text {. }
$$


Then

$$
Z \sinh \zeta=x \sinh \xi \cos \eta-y \cosh \xi \sin \eta+i(x \cosh \xi \sin \eta+y \sinh \xi \cos \eta) .
$$

The integral in (2) will converge if, along the path of integration,

$$
\operatorname{Re}[Z \sinh \zeta]=x \sinh \xi \cos \eta-y \cosh \xi \sin \eta>0 .
$$

This requirement is satisfied by

$$
-\frac{\pi}{2}<\arg Z+\epsilon<\frac{\pi}{2}
$$

where

$$
\epsilon \leq \tan ^{-1}\left[\frac{\tan \eta}{\tanh \xi}\right]
$$

Therefore, we can choose an appropriate path $L_{2}$ to define $H_{v}^{(2)}(Z)$ for

$$
\left(-\frac{\pi}{2}+\epsilon\right)<\arg Z<\frac{\pi}{2}-\epsilon
$$

which defines the half plane of convergence (see fig. II.1).

We now use the Fock expansion [Fock, 1945] with the substitution

$$
v=Z+\left(\frac{Z}{2}\right)^{1 / 3} t \text { and } \lambda=\left(\frac{Z}{2}\right)^{1 / 3} \zeta
$$

and the assumption that $t$ and $\lambda$ are finite and $|Z|$ is large.

This expansion gives

$$
H_{v}^{(2)}(Z) \cong-\frac{1}{\pi i} \int_{\Gamma} e^{t \lambda-\frac{\lambda^{3}}{3}}\left[1-\frac{1}{60}\left(\frac{Z}{2}\right)^{2 / 3} \lambda^{5}+\ldots\right] d \lambda
$$

where $\Gamma$ is an equivalent path of $L_{2}$ in the $\lambda$-plane as shown in figure II.2.

We now need to investigate the properties of the integral

$$
w(t)=\frac{1}{\sqrt{\pi}} \int_{\Gamma} e^{t \lambda-\frac{\lambda^{3}}{3}} d \lambda
$$

known as the Airy Integral.

The function $w(t)$ satisfies the differential equation

$$
w^{i i}(t)=t w(t)
$$

Figure II.1. Path of integration and half-plane of convergence for the integral of the Hankel function.
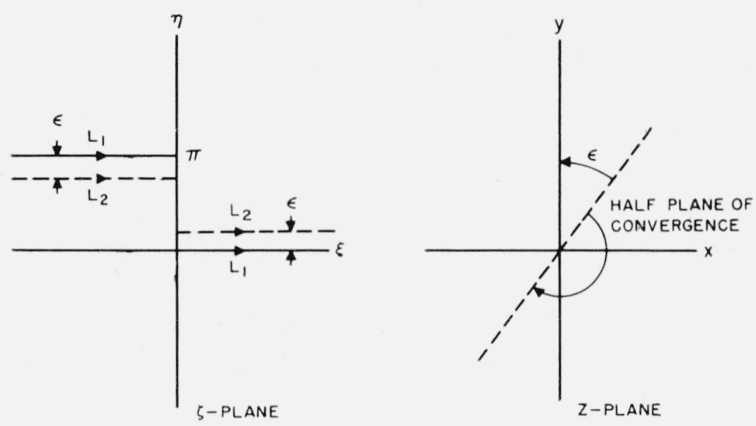


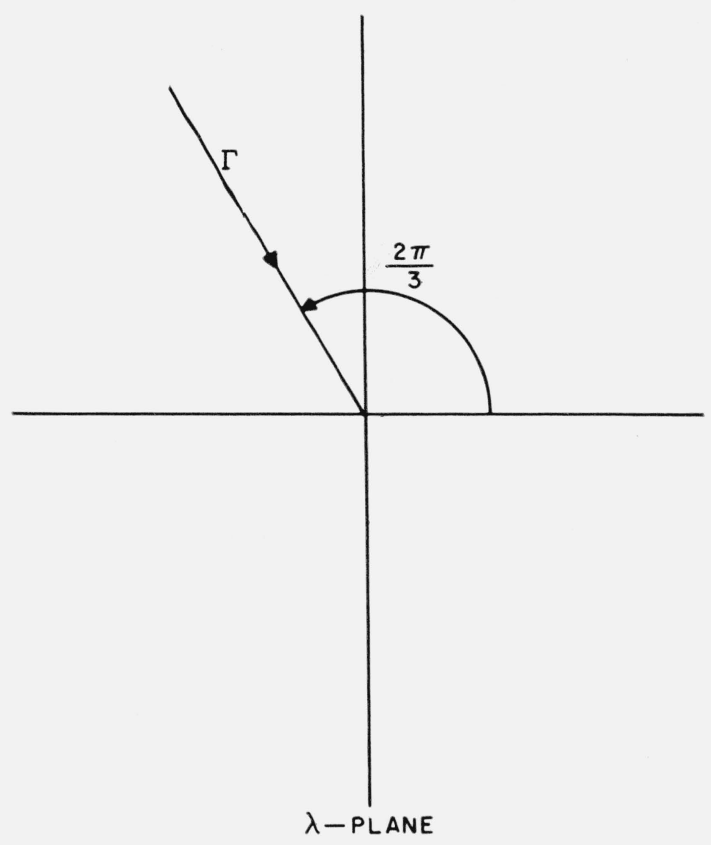

Figure II.2. Path of integration for the airy integral.

which leads to the recursion formulas:

$$
\begin{aligned}
& w^{i i i}(t)=w(t)+t w^{i}(t) \\
& w^{i v}(t)=2 w^{i}(t)+t w^{i i}(t)=2 w^{i}(t)+t^{2} w(t) \\
& w^{v}(t)=4 t w(t)+t^{2} w^{i}(t) .
\end{aligned}
$$

We also have

$$
w(t)=\sqrt{\frac{\pi}{3}} e^{-i \frac{2 \pi}{3}}(-t)^{1 / 2} H_{1 / 3}^{(2)}\left[\frac{2}{3}(-t)^{3 / 2}\right]
$$

where by $(-t)$ we mean $\left(t e^{i \pi}\right)$.

Using the relationship in (6), (7), and (8), we have the asymptotic expansion

$$
H_{v}^{(2)}(Z) \simeq-\frac{1}{i \sqrt{\pi}}\left(\frac{2}{Z}\right)^{1 / 3}\left[w(t)-\frac{t}{15}\left(\frac{2}{Z}\right)^{2 / 3} w(t)-\frac{t^{2}}{60}\left(\frac{2}{Z}\right)^{2 / 3} w^{i}(t)+\ldots\right] .
$$

The first term of the expansion in (10) represents the so-called "Hankel Approximation" and is used by several authors [Bremmer, 1949; Wait, 1956; Sensiper, 1957] in their residue series evaluations. Using (9) and the first term of (10) we have, as $Z \rightarrow \infty$

$$
H_{v}^{(2)}(Z) \cong \frac{i}{\sqrt{\pi}}\left(\frac{2}{Z}\right)^{1 / 3} w(t) \cong\left(\frac{2}{Z}\right)^{1 / 3} \sqrt{\frac{-t}{3}} e^{-i \pi / 6} H_{1 / 3}^{(2)}\left[\frac{2}{3}(-t)^{3 / 2}\right]
$$

where

$$
v-Z=\left(\frac{Z}{2}\right)^{1 / 3} t
$$

From (11) we have, for the derivative of the Hankel function

$$
H_{v}^{(2)^{\prime}}(Z) \cong \frac{i}{\sqrt{\pi}}\left(\frac{2}{Z}\right)^{1 / 3} w^{i}(t) \frac{\partial t}{\partial Z}
$$

But

$$
\frac{\partial t}{\partial Z} \simeq-\left(\frac{2}{Z}\right)^{1 / 3}
$$


Therefore

$$
H_{v}^{(2) \prime}(Z) \simeq \frac{1}{i \sqrt{\pi}}\left(\frac{2}{Z}\right)^{2 / 3} w^{i}(t)
$$

Using (9) in (12) gives

$$
H_{v}^{(2) '}(Z) \cong \frac{e^{-i \pi / 6}}{\sqrt{3}}\left(\frac{2}{Z}\right)^{2 / 3} t H_{2 / 3}^{(2)}\left[\frac{2}{3}(-t)^{3 / 2}\right]
$$

To complete this appendix, we investigate briefly the roots of $H_{v}^{(2)}(Z)=0$ and $H_{v}^{(2)}(Z)=0$. These roots, to first order, can be obtained from the approximations discussed above. For $Z$ large, such as $Z \geq 50$, the first order approximation for the roots is quite adequate. However, for moderate $Z$, higher order approximations might be necessary. Sensiper [1953 and 1957] discusses these roots quite thoroughly. At the end of this appendix we have, tabulated, the roots of $H_{v}^{(2) \prime}(Z)=0$, to first and second order, for values of $Z=10,15,20$ and 25 .

From the approximations in (11) and (12), we can find these roots, to a first order, quite easily. They correspond to the roots of

where

$$
H_{1 / 3}^{(2)}(\beta)=0 \text { and } H_{2 / 3}^{(2)}(\beta)=0
$$

$$
\beta=\frac{2}{3}(-t)^{3 / 2} .
$$

From Watson [1952] we have the identity

$$
H_{1 / 3}^{(2)}(\beta)=\frac{i}{\sin \pi / 3}\left[J_{-1 / 2}(\beta)-e^{\frac{i \pi}{3}} J_{1 / 3}(\beta)\right] .
$$

If we let $\beta=x e^{i \pi}$ and use the identity

$$
J_{v}\left(x e^{i \pi}\right)=e^{i v \pi} J_{v}(x)
$$

then (14) becomes

$$
H_{1 / 3}^{(2)}(\beta)=\frac{i e^{-i \frac{\pi}{3}}}{\sin \pi / 3}\left[J_{-1 / 3}(x)+J_{1 / 3}(x)\right] .
$$

Therefore, to determine the roots $v_{m 1}$ of $H_{v}^{(2)}(Z)=0$ to the first order, we need to determine the roots of

$$
J_{-1 / 3}(x)+J_{1 / 3}(x)=0 .
$$

The roots $x_{m 1}$ of (17) are well known and we have a one to one correspondence between $v_{m 1}$ and $x_{m_{1}}$.

$$
\begin{aligned}
& v_{m 1}=Z+\left(\frac{Z}{2}\right)^{1 / 3} t_{m 1} \\
& \beta_{m 1}=x_{m 1} e^{i \pi}=\frac{2}{3}\left(-t_{m 1}\right)^{3 / 2} \\
& t_{m 1}=\left(\frac{3 x_{m 1}}{2}\right)^{2 / 3} e^{-i \pi / 3} .
\end{aligned}
$$

The roots of $\mathrm{H}_{2 / 3}^{(2)}(\beta)=0$ can be approximated in a similar manner. We have

$$
H_{2 / 3}^{(2)}(\beta)=\frac{i}{\sin \frac{2 \pi}{3}}\left[J_{-2 / 3}(x)-e^{\frac{i 2 \pi}{3}} J_{2 / 3}(\beta)\right] .
$$


Letting $\beta=x e^{i \pi}$ again and using the identity in (15) we have for (19)

$$
H_{2 / 3}^{(2)}\left(x e^{i \pi}\right)=\frac{i e^{-i \frac{2 \pi}{3}}}{\sin \frac{2 \pi}{3}}\left[J_{-2 / 3}(x)-J_{2 / 3}(x)\right] .
$$

Therefore, to determine the roots $v_{m 2}$ of $H_{v}^{(2) '}(Z)$ to first order, we first need to know the roots of

$$
J_{-2 / 3}(x)-J_{2 / 3}(x)=0 .
$$

The roots $x_{m 2}$ of (21) are well known and we again have a one to one correspondence between $v_{m 2}$ and $x_{m 2}$.

$$
\begin{aligned}
& v_{m 2}=Z+\left(\frac{Z}{2}\right)^{1 / 3} t_{m 2} \\
& \beta_{m 2}=x_{m 2} e^{i \pi}=\left(\frac{2}{3}\right)\left(-t_{m 2}\right)^{3 / 2} \\
& t_{m 2}=\frac{3 x_{m 2}}{2}{ }^{2 / 3} e^{-i \pi / 3} .
\end{aligned}
$$

Sensiper [1957] in his discussion of these roots, gives the following expansions.

$$
\begin{aligned}
& \frac{v_{m 1}}{Z}=1+\frac{1}{2}\left(\frac{2}{Z}\right)^{2 / 3} t_{m 1}+\frac{1}{120}\left(\frac{2}{Z}\right)^{4 / 3} t_{m 1}^{2}+\ldots \\
& \frac{v_{m 2}}{Z}=1+\frac{1}{2}\left(\frac{2}{Z}\right)^{2 / 3} t_{m 2}+\frac{1}{120}\left(\frac{2}{Z}\right)^{4 / 3}\left(1+\underset{3 x_{m 2}^{2}}{8}\right) t_{m 2}^{2}+\ldots
\end{aligned}
$$

\begin{tabular}{|c|c|c|c|c|}
\hline$m$ & $x_{m 1}$ & $x_{m 2}$ & $t_{m i} e^{i \pi / 3}$ & $t_{m 2} e^{i \pi / 3}$ \\
\hline $\begin{array}{l}1 \\
2 \\
3 \\
4 \\
5\end{array}$ & $\begin{array}{r}2.383 \\
5.512 \\
8.647 \\
11.787 \\
14.927\end{array}$ & $\begin{array}{r}0.686 \\
3.902 \\
7.058 \\
10.202 \\
13.348\end{array}$ & $\begin{array}{l}2.338 \\
\text { 4. } 099 \\
5.358 \\
6.787 \\
7.944\end{array}$ & $\begin{array}{l}\text { 1. } 019 \\
\text { 3. } 248 \\
\text { 4. } 820 \\
6.163 \\
\text { 7. } 372\end{array}$ \\
\hline
\end{tabular}

\begin{tabular}{|c|c|c|c|c|}
\hline \multirow[b]{3}{*}{$\begin{array}{l}1 . \\
2 \\
3\end{array}$} & \multicolumn{2}{|c|}{$v_{m 2} / Z$ for $Z=10$} & \multicolumn{2}{|c|}{$v_{m 2} / Z$ from $Z=15$} \\
\hline & By eq (22) & By eq (24) & By eq (22) & By eq (24) \\
\hline & $\begin{array}{l}1.09-i 0.161 \\
1.28-i 0.481 \\
1.41-i 0.714 \\
1.53-i 0.913 \\
1.63-i 1.09\end{array}$ & $\begin{array}{l}\text { 1. } 09-i 0.168 \\
1.27-i 0.491 \\
1.40-i 0.693 \\
1.51-i 0.946 \\
1.60-i 1.14\end{array}$ & $\begin{array}{l}1.071-i 0.123 \\
1.211-i 0.366 \\
1.313-i 0.543 \\
1.401-i 0.694 \\
1.479-i 0.830\end{array}$ & $\begin{array}{l}\text { 1. } 069-i 0.127 \\
\text { 1. } 208-i 0.372 \\
\text { 1. } 306-i 0.555 \\
\text { 1. } 390-i 0.713 \\
1.466-i 0.857\end{array}$ \\
\hline & \multicolumn{2}{|c|}{$v_{m 2} / Z$ for $Z=20$} & \multicolumn{2}{|c|}{$v_{m 2} / Z$ for $Z=25$} \\
\hline & By eq (22) & By eq (24) & By eq (22) & By eq (24) \\
\hline $\begin{array}{l}1 \\
2 \\
3 \\
4 \\
5\end{array}$ & $\begin{array}{l}1.059-i 0.102 \\
1.175-i 0.304 \\
1.260-i 0.451 \\
1.333-i 0.576 \\
1.398-i 0.689\end{array}$ & $\begin{array}{l}1.051-i 0.105 \\
1.173-i 0.308 \\
1.255-i 0.459 \\
1.325-i 0.589 \\
1.387-i 0.707\end{array}$ & $\begin{array}{l}\text { 1. } 051-i 0.0878 \\
1.151-i 0.262 \\
1.224-i 0.388 \\
1.287-i 0.496 \\
1.343-i 0.594\end{array}$ & $\begin{array}{l}\text { 1. } 050-i 0.0895 \\
1.149-i 0.265 \\
1.220-i 0.394 \\
\text { 1. } 281-i 0.506 \\
1.335-i 0.608\end{array}$ \\
\hline
\end{tabular}

Table II. 1 is a list of the roots, $x_{m}$ and $t_{m}$, and table II.2 shows a comparison of the values of $v_{m 2} / Z$ computed by (22) and (24) for several values of $Z$.

TABLE II.1. Table of roots related to the roots of the Hankel functions

TABLE II.2. Comparison of values of $v_{m 2} / Z$ computed by equations (22) and (24) 


\section{References}

Bremmer, H., Terrestrial radio waves (Elsevier Publishing Co., 1949).

Courant, R., and D. Hilbert, Methods of mathematical physics, Vol. I (Interscience Publishers, 1953).

Fock, V. A., Diffraction of radio waves around the earth's surface, J. Phys. USSR 9, pp. $255-266$ (1945).

Held, G., and G. Hasserjian, An experimental analysis of the surface and tip excitation of a cone by a slot, University of Washington Tech. Rept. No. 25 (March 1958).

Jeffreys, H., and B. S. Jeffreys, Methods of mathematical physics, 3d ed. (Cambridge, 1956).

Lucke, W. S., Mutual admittance of slots in cylinders, Tech. Rept. No. 36, Stanford Research Institute, Stanford, Calif. (Feb. 1953).

Marcuvitz, N., and J. Schwinger, On the representation of the electric and magnetic fields produced by currents and discontinuities in wave guides, J. Appl. Phys. 22, No. 6, 806-819 (June 1951).

Nishida, S., Coupled leaky waveguides II: Two parallel slits in a cylinder, IRE Trans. AP-8, No."4, 354-360 (July 1960).

Oliner, A. A., Impedance properties of narrow radiating slots in the broad face of a rectangular waveguide, IRE Trans. AP-5, No. 1, 12-20 (Jan. 1957).

Papas, C. H., and R. King, Currents on the surface of an infinite cylinder excited by an axial slot, Quart." Appl. Math. \%, No. 2, 175-182 (1949).

Sensiper, S., Cylindrical radio waves, Hughes Aircraft Co. Research and Development Labs., T.M. No. 310 (June 1953).

Sensiper, S., Cylindrical radio waves, IRE Trans. AP-5 (1957).

Silver, S., and W. K. Saunders, The external field produced by a slot on an infinite circular cylinder, J. Appl. Phys. 21, No. 2, 153-158 (Feb. 1950).

Wait, J. R., Currents excited on a conducting surface of large radius of curvature, IRE Trans. MTT-4, No. 3, 144-145 (July 1956).

Wait, J. R., Electromagnetic radiation from cylindrical structures (Pergamon Press, New York and London, 1959).*

Watson, G. N., Theory of Bessel functions, 2d ed. (Cambridge, 1952).

Wittaker, E. T., and G. N. Watson, Modern Analysis, 4th ed. (Cambridge 1952).

${ }^{*}$ J. R. Wait has supplied the following errata in his monograph: On p. 64 , eq $(202 \mathrm{~b}),(2 / x)^{1 / 3} \operatorname{should}$ be $(2 / x)^{2 / 3}$. On p. 129, eq $(351), F_{m}(k 0$ sin $\theta)$ should be $F_{m}\left(k_{0} \cos \theta\right)$. Here pole contributions can be neglected since $k \rho>>1$ as stated.

(Paper 66D3-201) 